\title{
Time-like reductions of five-dimensional supergravity
}

\author{
V. Cortés, ${ }^{a}$ P. Dempster ${ }^{b}$ and T. Mohaupt ${ }^{b}$ \\ ${ }^{a}$ Department of Mathematics and Center for Mathematical Physics, University of Hamburg \\ Bundesstraße 55, D-20146 Hamburg, Germany \\ ${ }^{b}$ Department of Mathematical Sciences, University of Liverpool, \\ Peach Street, Liverpool L69 7ZL, U.K. \\ E-mail: cortes@math.uni-hamburg.de, pdemp@liverpool.ac.uk, \\ Thomas. Mohaupt@liv.ac.uk
}

ABSTRACT: In this paper we study the scalar geometries occurring in the dimensional reduction of minimal five-dimensional supergravity to three Euclidean dimensions, and find that these depend on whether one first reduces over space or over time. In both cases the scalar manifold of the reduced theory is described as an eight-dimensional Lie group $L$ (the Iwasawa subgroup of $G_{2(2)}$ ) with a left-invariant para-quaternionic-Kähler structure. We show that depending on whether one reduces first over space or over time, the group $L$ is mapped to two different open $L$-orbits on the pseudo-Riemannian symmetric space $G_{2(2)} /(\mathrm{SL}(2) \cdot \mathrm{SL}(2))$. These two orbits are inequivalent in the sense that they are distinguished by the existence of integrable $L$-invariant complex or para-complex structures.

KEYwords: Differential and Algebraic Geometry, Supergravity Models

ARXIV EPRINT: 1401.5672 


\section{Contents}

1 Introduction 1

2 Dimensional reduction of pure five-dimensional supergravity 4

3 The group $G_{2(2)}$, its Iwasawa subgroup, and the symmetric space $S=$ $G_{2(2)} /(\mathrm{SL}(2) \cdot \mathrm{SL}(2))$

3.1 The noncompact group of type G2 9

3.2 The symmetric para-quaternionic-Kähler manifold $S=G_{2(2)} /\left(\mathrm{SL}_{2} \cdot \mathrm{SL}_{2}\right) \quad 9$

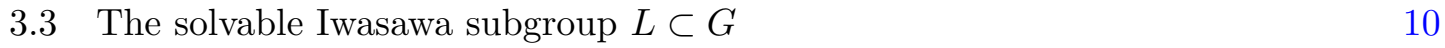

4 Realization of the scalar manifolds of the reduced theories as open orbits

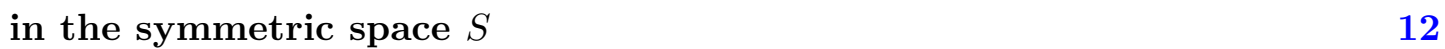

4.1 Iwasawa subgroups of $G$ with an open orbit on $S \quad 12$

$\begin{array}{ll}4.2 \text { Automorphisms of the solvable algebra } & 14\end{array}$

$\begin{array}{lll}4.3 & \text { Identifying the open orbit corresponding to Time-Space reduction } & 17\end{array}$

4.4 Identifying the open orbit corresponding to Space-Time reduction 18

5 Geometric structures on the Iwasawa subgroup of $G_{2(2)} \quad 20$

5.1 Computation of the Levi Civita connection 22

5.2 Curvature 24

5.3 Relation with $Q$-representations of Kählerian Lie algebras in the Riemannian case 24

5.4 Conjugate Iwasawa subgroups vs disjoint open $L$-orbits 26

\section{Introduction}

The dimensional reduction of gravity, supergravity and string theory over time reveals symmetries that are otherwise hidden, is relevant for gravitational instantons, and allows one to generate stationary solutions by subsequent dimensional lifting [1-4]. In the simplest examples the scalar manifolds of theories obtained by dimensional reduction on tori of Lorentzian signature are locally symmetric Riemannian spaces with split signature. Particular cases studied in the literature are the symmetric spaces occurring when gravity coupled to matter is reduced from four to three dimensions [2]; reductions of $D$-dimensional gravity, of bosonic and heterotic string theory, and of eleven-dimensional supergravity on Lorentzian tori [5]; and reductions of extended four-dimensional supergravities with symmetric target spaces over a time-like circle [6].

Global aspects of time-like reductions have been less studied, but some complications have been observed in toroidal compactifications of string theory which include a time-like 
direction [3]. While in space-like reductions leading to Riemannian symmetric target spaces $M=G / K$ of non-compact type one can rely on the Iwasawa decomposition $G=K L$, to provide a global parametrization of $M$ using the simply transitive action of the solvable Iwasawa subgroup $L \subset G$, such a global parametrization is no longer possible for the pseudo-Riemannian symmetric spaces $G / H$ appearing in time-like reductions. However examples show that it might still be possible to find a decomposition of the form $H L$ for an open subset of $U \subset G$, leading to a local parametrization of the space $G / H$. In this case the Iwasawa subgroup $L$ still acts with an open orbit. In [7] it was shown that duality transformations relating BPS to non-BPS solutions correspond to 'singular' elements of $G$, i.e. elements outside an open dense set $U \subset G$ decomposed as $U=H L$. In [8] it was shown that solutions with regular event horizons correspond to complete geodesics which are contained within a 'solv-patch', i.e. an open orbit of the Iwasawa subgroup, whereas geodesics which are not fully contained in a single solv-patch lift to singular space-time geometries.

In this paper we investigate further consequences of the non-transitive action of the Iwasawa subgroup. If the Iwasawa subgroup acts with more than one open orbit, then there is no a priori reason why any two open orbits should be equivalent. And if open orbits are not equivalent, it becomes necessary to decide which orbit corresponds to a given dimensional reduction. More specifically, we will now explain why this becomes an issue when reducing five-dimensional supergravity coupled to vector multiplets to three Euclidean dimensions. Recall that the dimensional reduction of four-dimensional $N=2$ vector multiplets to three Lorentzian dimensions leads to a scalar geometry which is quaternionic-Kähler [9]. The resulting map between (projective) special Kähler manifolds and quaternionic-Kähler manifolds is known as the c-map. This result extends Alekseevsky's construction [10] of symmetric and non-symmetric quaternionic-Kähler manifolds with a simply transitive solvable group of isometries from certain Kähler manifolds, see also [11-13]. One of the simplest examples described by Alekseevsky is the symmetric quaternionic-Kähler manifold $G_{2(2)} / \mathrm{SO}(4)$ presented as a solvable group with left-invariant quaternionic-Kähler structure. This manifold comprises the universal sector of five-dimensional supergravity reduced to three dimensions. The Alekseevsky spaces come equipped with an integrable complex structure compatible with the quaternionic structure. More recently it was shown in [14] that this is even true for all $c$-map spaces.

If $N=2$ vector multiplets are dimensionally reduced with respect to time, the target space geometry is expected to be para-quaternionic-Kähler instead of quaternionic-Kähler, as explained in [15]. Recall that a pseudo-Riemannian manifold $(M, g)$ of dimension $4 n>4$ is called para-quaternionic-Kähler if its holonomy group is a subgroup of $\operatorname{Sp}\left(\mathbb{R}^{2}\right) \cdot \operatorname{Sp}\left(\mathbb{R}^{2 n}\right) \subset$ $\mathrm{SO}(2 n, 2 n)$ [16]. Geometrically this means that the manifold $(M, g)$ admits a parallel subbundle $Q \subset \operatorname{End}(T M)$ which is point-wise spanned by three anti-commuting skewsymmetric endomorphisms $I, J, K=I J$ such that $I^{2}=J^{2}=-K^{2}=\mathrm{Id}$.

In a forthcoming paper [17] we prove that both the dimensional reduction of $N=2$ supergravity with vector multiplets over time and the dimensional reduction of Euclidean $N=2$ supergravity with vector multiplets over space results in scalar target spaces that are para-quaternionic-Kähler. Moreover, while in the first case the para-quaternionic structure 
contains an integrable complex structure, it contains an integrable para-complex structure in the second case. This indicates that when starting in five dimensions and reducing over time and one space-like dimension, the result will depend on the order in which the reductions are taken. Since this is an unexpected result, we will in this paper investigate the simplest case, the dimensional reduction of pure five-dimensional supergravity, in detail. We emphasize that, while our work is motivated by [17], this paper is completely selfcontained.

The dimensional reduction of pure five-dimensional supergravity with respect to time and one space-like dimension leads to a scalar target space which is locally isometric to the symmetric space

$$
G_{2(2)} / S O_{0}(2,2) \simeq G_{2(2)} /(\mathrm{SL}(2) \cdot \mathrm{SL}(2))
$$

[18-20], which is para-quaternionic-Kähler. The classification of symmetric paraquaternionic-Kähler manifolds of non-zero scalar curvature follows from the fact that the isometry group of such a space is simple, see Theorem 5 of [16], together with Berger's classification of pseudo-Riemannian symmetric spaces of semi-simple groups [21, 22]. The resulting list can be found in $[23,24]$ and contains the space (1.1). This space represents the universal sector of the reduction of five-dimensional supergravity coupled to matter. In general, the spaces obtained by such reductions will neither be symmetric, nor even homogeneous. The dimensional reduction of five-dimensional supergravity with an arbitrary number of vector multiplets to three Euclidean dimensions will be investigated in a future publication [25].

The space (1.1) has been studied in the literature in the context of generating stationary solutions in four and five dimensions, in particular stationary four-dimensional black holes $[4,19]$ and black string solutions of five-dimensional supergravity [26, 27]. In [26] it was verified that one obtains locally isometric locally symmetric spaces irrespective of whether the reduction is carried out first over space or first over time. It was shown in [4] that these two reductions are related to the purely space-like reduction by analytic continuation, see further comments in section 2. In this paper we will make precise the relation between the corresponding scalar manifolds and open orbits of the Iwasawa subgroup $L$ of $G_{2(2)}$ on $G_{2(2)} /(\mathrm{SL}(2) \cdot \mathrm{SL}(2))$. We will show that while the scalar manifolds are locally isometric they are not related by an automorphism of $L$, and are geometrically distinguished by the integrability properties of the left-invariant almost complex and para-complex structures within the para-quaternionic structure.

Let us next give a more detailed summary of the results obtained in this paper. We perform the dimensional reduction of pure five-dimensional supergravity to three Euclidean dimensions and find that the resulting scalar geometry is naturally described as a solvable Lie group $L^{\left(\epsilon_{1}, \epsilon_{2}\right)}$ endowed with a left-invariant pseudo-Riemannian metric $g^{\left(\epsilon_{1}, \epsilon_{2}\right)}$ of split signature. The parameters $\epsilon_{1}, \epsilon_{2} \in\{1,-1\}$ indicate whether the reduction is over a spacelike $(\epsilon=-1)$ or over a time-like $(\epsilon=1)$ direction in the subsequent reduction steps. For comparison we will also review the case of a purely space-like reduction $\left(\epsilon_{1}=\epsilon_{2}=-1\right)$. We find that all three groups $L^{\left(\epsilon_{1}, \epsilon_{2}\right)}$ are isomorphic to the solvable Iwasawa subgroup of $G_{2(2)}$, which we will denote by $L$. In contrast to this, we prove that the metrics $g^{(1,-1)}$ 
and $g^{(-1,1)}$ are not related by an automorphism of the group $L$. However, we show that both pseudo-Riemannian manifolds $\left(L, g^{(1,-1)}\right)$ and $\left(L, g^{(-1,1)}\right)$ can be mapped by a $\phi$ equivariant (respectively, $\phi^{\prime}$-equivariant) isometric covering to open orbits

$$
M=\phi(L) \cdot o, M^{\prime}=\phi^{\prime}(L) \cdot o \subset S=G / H=G_{2(2)} /(\mathrm{SL}(2) \cdot \mathrm{SL}(2)),
$$

respectively, where $\phi, \phi^{\prime}: L \rightarrow G_{2(2)}$ are embeddings of $L$ into $G_{2(2)}$ and $o=e H$ is the canonical base point of the pseudo-Riemannian symmetric space $\left(S=G / H, g_{S}\right)$. This proves that the pseudo-Riemannian manifolds $\left(L, g^{(1,-1)}\right)$ and $\left(L, g^{(-1,1)}\right)$ are locally symmetric and locally isometric to each other.

The symmetric space $\left(S, g_{S}\right)$ carries a canonical compatible $G$-invariant paraquaternionic structure $Q$, which we will explicitly describe in section 3.2. This provides a direct proof that $\left(S, g_{S}, Q\right)$ is a para-quaternionic-Kähler manifold, as well as the open orbits $M, M^{\prime} \subset S$. Pulling back the para-quaternionic structure $Q$ by the local isometries $\phi:\left(L, g^{(1,-1)}\right) \rightarrow M, \phi^{\prime}:\left(L, g^{(-1,1)}\right) \rightarrow M^{\prime}$ we obtain left-invariant para-quaternionicKähler structures $\left(g^{(1,-1)}, Q^{(1,-1)}\right)$ and $\left(g^{(-1,1)}, Q^{(-1,1)}\right)$ on $L$. We show that $Q^{(1,-1)}$ contains a left-invariant integrable para-complex structure $J_{1}=J_{1}^{(1,-1)}$, whereas $Q^{(-1,1)}$ contains a left-invariant integrable complex structure $J_{1}=J_{1}^{(-1,1)}$. The structure $J_{1}$ is included in a standard basis $\left(J_{1}, J_{2}, J_{3}\right)$ of $Q^{\left(\epsilon_{1}, \epsilon_{2}\right)}$, which we specify explicitly on the Lie algebra $\mathfrak{l}$ of $L$.

The left-invariant structure $J_{1}$ is not the only left-invariant complex $\left(\epsilon_{1}=-1\right)$ or paracomplex $\left(\epsilon_{1}=1\right)$ structure on $L$ which is integrable and skew-symmetric. We explicitly describe a second such structure $\tilde{J}_{1}$, commuting with $J_{1}$, which does not belong to the (para-)quaternionic structure.

Finally we calculate the Levi-Civita connection and curvature tensor of the metrics $g^{\left(\epsilon_{1}, \epsilon_{2}\right)}$, in terms of a basis of left-invariant vector fields on $L$. Using these formulae we give a second proof of the fact that the metrics $g^{\left(\epsilon_{1}, \epsilon_{2}\right)}$ are locally symmetric and paraquaternionic-Kähler by checking that the covariant derivative of the curvature tensor vanishes, and that $Q^{\left(\epsilon_{1}, \epsilon_{2}\right)}$ is parallel.

\section{Dimensional reduction of pure five-dimensional supergravity}

In this section we perform the dimensional reduction of pure five-dimensional supergravity to three dimensions. The reductions over two space-like dimensions and over one space-like and one time-like dimension will be considered in parallel. In the latter case the time-like reduction can be either taken as the first or the second step. We will be interested in comparing both options to one another.

We start with the action for five-dimensional supergravity, coupled to an arbitrary number $n_{V}^{(5)}$ of vector multiplets. In the conventions of [28], the bosonic part of the action takes the following form:

$$
\begin{gathered}
S_{5}=\int d^{5} x\left[\sqrt{\hat{g}}\left(\frac{\hat{R}}{2}-\frac{3}{4} a_{i j} \partial_{\hat{\mu}} h^{i} \partial^{\hat{\mu}} h^{j}-\frac{1}{4} a_{i j} \mathcal{F}_{\hat{\mu} \hat{\nu}}^{i} \mathcal{F}^{j \mid \hat{\mu} \hat{\nu}}\right)\right. \\
\left.+\frac{1}{6 \sqrt{6}} c_{i j k} \varepsilon^{\hat{\mu} \hat{\nu} \hat{\rho} \hat{\sigma} \hat{\lambda}} \mathcal{F}_{\hat{\mu} \hat{\nu}}^{i} \mathcal{F}_{\hat{\rho} \hat{\sigma}}^{j} \mathcal{A}_{\hat{\lambda}}^{k}\right] .
\end{gathered}
$$


Here $\hat{\mu}, \hat{\nu}, \ldots$ are five-dimensional Lorentz indices and $i=0,1, \ldots, n_{V}^{(5)}$ labels the fivedimensional gauge fields. The scalars $h^{i}$ are understood to satisfy the constraint

$$
\mathcal{V}=c_{i j k} h^{i} h^{j} h^{k}=1
$$

where $\mathcal{V}$ is a prepotential which encodes all the couplings. While we will analyse the dimensional reduction of five-dimensional supergravity with vector multiplets in a separate paper [25], in this article we will only consider the case of pure supergravity, where $\mathcal{V}=$ $\left(h^{0}\right)^{3}=1$. Then the bosonic action (2.1) reduces to the one of Einstein-Maxwell theory supplemented by a Chern-Simons term:

$$
S=\int d^{5} x\left[\sqrt{\hat{g}}\left(\frac{\hat{R}}{2}-\frac{1}{4} \mathcal{F}_{\hat{\mu} \hat{\nu}} \mathcal{F}^{\hat{\mu} \hat{\nu}}\right)+\frac{1}{6 \sqrt{6}} \varepsilon^{\hat{\mu} \hat{\nu} \hat{\rho} \hat{\sigma} \hat{\lambda}} \mathcal{F}_{\hat{\mu} \hat{\nu}} \mathcal{F}_{\hat{\rho} \hat{\sigma}} \mathcal{A}_{\hat{\lambda}}\right] .
$$

We perform the dimensional reduction over 2 directions by taking the metric ansatz $M_{5}=$ $S^{1} \times S^{1} \times M_{3}$ with

$$
d s_{(5)}^{2}=-\epsilon_{1} e^{2 \sigma}\left(d x^{0}+\mathcal{A}^{0}\right)^{2}-\epsilon_{2} e^{2 \phi-\sigma}\left(d x^{4}+B\right)^{2}+e^{-2 \phi-\sigma} d s_{(3)}^{2},
$$

where $\epsilon_{1,2}$ take the values -1 for reduction over a space-like direction and +1 for a time-like reduction ${ }^{1}$. We also introduce the variable $\epsilon:=-\epsilon_{1} \epsilon_{2}=(-1)^{t}$, where $t$ is the number of time-like directions in the three-dimensional theory. Note that we can take either $x^{0}$ or $x^{4}$ to be time-like. There are two Kaluza-Klein vectors: the four-dimensional vector $\mathcal{A}^{0}$ arising from the first reduction step and the three-dimensional vector $B$ arising from the second. It will be convenient to refer to the three different reductions as SS-type (spacelike/space-like, $\epsilon_{1}=\epsilon_{2}=-1$ ), ST-type (space-like/time-like, $\epsilon_{1}=-1, \epsilon_{2}=1$ ) and TS-type (time-like/space-like, $\epsilon_{1}=1, \epsilon_{2}=-1$ ).

After reduction, we obtain the following three-dimensional Lagrangian:

$$
\begin{aligned}
\mathcal{L}_{3}= & \frac{R}{2}+\frac{3}{4 y^{2}} \epsilon_{1}(\partial x)^{2}-\frac{3}{4 y^{2}}(\partial y)^{2}-\frac{1}{4 \phi^{2}}(\partial \phi)^{2}+\frac{1}{4 \phi^{2}} \epsilon_{1}\left(\partial \tilde{\phi}+p^{I} \overleftrightarrow{\partial} s_{I}\right)^{2} \\
& +\frac{y^{3}}{12 \phi} \epsilon\left(\partial p^{0}\right)^{2}+\frac{y}{4 \phi} \epsilon_{2}\left(\partial p^{1}-x \partial p^{0}\right)^{2} \\
& +\frac{3}{y^{3} \phi} \epsilon_{2}\left(\partial s_{0}+x \partial s_{1}-\frac{1}{6} x^{3} \partial p^{0}+\frac{1}{2} x^{2} \partial p^{1}\right)^{2} \\
& +\frac{1}{y \phi} \epsilon\left(\partial s_{1}-\frac{1}{2} x^{2} \partial p^{0}+x \partial p^{1}\right)^{2} .
\end{aligned}
$$

Here $R$ is the three-dimensional Ricci scalar which does not give rise to local dynamics. The dynamical fields are the eight scalar fields $x, y, \phi, \tilde{\phi}, p^{0}, p^{1}, s_{0}, s_{1}$, which have the following five-dimensional origin: the scalars $x$ and $y$ arise by dimensional reduction from five to four dimensions, and encode the degrees of freedom corresponding to the Kaluza-Klein scalar $\sigma$ and the component $\mathcal{A}_{0}$ of the five-dimensional vector field $\mathcal{A}$. Explicitly, we have

$$
y=6^{\frac{1}{3}} e^{\sigma} h^{0}, \quad x=2 \cdot 6^{-\frac{1}{6}} \mathcal{A}_{0} .
$$

\footnotetext{
${ }^{1}$ Note that other papers studying reduction with respect to time, including [26, 29], use the opposite sign convention.
} 
Following the procedure of [28] we have absorbed the Kaluza Klein scalar $\sigma$ into $h^{0}$ to obtain scalars fitting into four-dimensional vector multiplets. In this formulation $x$ and $y$ are independent dynamical scalar fields, whereas $\sigma$ is a dependent field which can be expressed in terms of $y$ via $e^{\sigma}=6^{-1 / 3} y$.

The scalars $\phi$ and $\tilde{\phi}$ arise from reducing the space-time metric from four to three dimensions. The field $\phi$ appearing in (2.4) is related to the Kaluza-Klein scalar in our ansatz (2.3) via $e^{2 \phi} \rightarrow \phi$, while $\tilde{\phi}$ arises from dualizing the Kaluza-Klein vector. In particular,

$$
H_{m n}=\frac{1}{\phi^{2}} \epsilon_{m n p}\left(\partial^{p} \tilde{\phi}+p^{0} \overleftrightarrow{\partial}^{p} s_{0}+p^{1} \overleftrightarrow{\partial^{p}} s_{1}\right)
$$

where $H_{m n}=2 \partial_{[m} B_{n]}$ is the field strength associated with the second Kaluza-Klein vector.

After reduction from five to four dimensions, we have two vector fields, namely the reduction of the five-dimensional vector field and the Kaluza-Klein vector $\mathcal{A}^{0}$. Upon reduction to three dimensions, each gives rise to 2 scalars: $p^{0}$ and $p^{1}$ correspond to the four-dimensional components of the two vector fields,

$$
\mathcal{A}_{4}^{0}=-\sqrt{2} p^{0}, \quad \mathcal{A}_{4}=\frac{6^{\frac{1}{6}}}{\sqrt{2}}\left(p^{1}-x p^{0}\right),
$$

while $s_{0}$ and $s_{1}$ are obtained by dualizing the vector fields after reduction to three dimensions:

$$
\begin{aligned}
\mathcal{F}_{m n}^{0}= & 2 \sqrt{2} B_{[m} \partial_{n]} p^{0}-\epsilon \frac{6 \sqrt{2}}{\phi y^{3}} \epsilon_{m n p}\left(\partial^{p} s_{0}+x \partial^{p} s_{1}+\frac{1}{2} x^{2} \partial^{p} p^{1}-\frac{1}{6} x^{3} \partial^{p} p^{0}\right), \\
\mathcal{F}_{m n}= & \frac{6^{\frac{1}{6}}}{\sqrt{2}}\left\{\begin{array}{l}
-2 B_{[m}\left(\partial_{n]} p^{1}-x \partial_{n]} p^{0}\right) \\
\left.\quad+\frac{2 \epsilon_{2}}{\phi y} \epsilon_{m n p}\left(\partial^{p} s_{1}+x \partial^{p} p^{1}-\frac{1}{2} x^{2} \partial^{p} p^{0}\right)+\sqrt{2} \mathcal{A}_{[m}^{0} \partial_{n]} x\right\} .
\end{array}\right.
\end{aligned}
$$

The scalar manifolds obtained by SS-, ST- and TS-reduction are denoted $M^{(S S)}, M^{(S T)}$ and $M^{(T S)}$ respectively, and the corresponding metrics are denoted $g^{(S S)}=g^{(-1,-1)}, g^{(S T)}=$ $g^{(-1,1)}$ and $g^{(T S)}=g^{(1,-1)}$, respectively.

It is known that in the reduction over two space-like directions the eight scalars parametrize the symmetric space $G_{2(2)} / \mathrm{SO}(4)$, which is quaternionic-Kähler. Here $G_{2(2)}$ denotes the non-compact real form of the exceptional Lie group of type $G 2$. It is also known that the reduction over one space-like and one time-like dimension gives rise to a space which is locally isometric to the pseudo-Riemannian symmetric space $G_{2(2)} /(\mathrm{SL}(2) \cdot \mathrm{SL}(2))$, which is para-quaternionic-Kähler, as expected for three-dimensional Euclidean hypermultiplets [15]. From (2.4) it is not manifest that reduction over time followed by reduction over space $\left(\epsilon_{1}=1, \epsilon_{2}=-1\right)$ results in the same manifold as when reducing in the opposite order $\left(\epsilon_{1}=-1, \epsilon_{2}=1\right)$. It is however clear that both reductions are related to the purely space-like reduction $\epsilon_{1}=\epsilon_{2}=-1$, and hence to one another, by analytic continuation, since $G_{2(2)} / \mathrm{SO}(4)$ and $G_{2(2)} /(\mathrm{SL}(2) \cdot \mathrm{SL}(2))$ are real forms of the same complex-Riemannian symmetric space $G_{2}^{\mathbb{C}} / \mathrm{SO}(4, \mathbb{C})$. The analytic continuations between the SS-reduction and the TS-reduction and ST-reduction for the more general case including an arbitrary number of 
vector multiplets were given explicitly in [4]. Restricting to pure supergravity, and using our conventions, the continuation from the SS-reduction to the TS-reduction takes the form

$$
\left(y, x, \phi, \tilde{\phi}, p^{0}, p^{1}, s_{0}, s_{1}\right) \mapsto\left(y, i x, \phi, i \tilde{\phi},-i p^{0}, p^{1},-s_{0}, i s_{1}\right),
$$

whilst the continuation from the SS-reduction to the ST-reduction takes the form

$$
\left(y, x, \phi, \tilde{\phi}, p^{0}, p^{1}, s_{0}, s_{1}\right) \mapsto\left(y, x, \phi,-\tilde{\phi}, i p^{0}, i p^{1}, i s_{0}, i s_{1}\right) .
$$

It is straightforward to check that these substitutions change the relative signs of terms in (2.4) in precisely the same way as making the corresponding changes of the parameters $\epsilon_{1}$ and $\epsilon_{2}$. The authors of [4] also specify a map relating the ST- and TS-reductions in their formulae (3.16)-(3.20). A different approach was taken in [26], where the parametrization of the scalar fields induced by dimensional reduction was related to a standard parametrization of the symmetric space $G_{2(2)} /(\mathrm{SL}(2) \cdot \mathrm{SL}(2))$. We will use a different parametrization which allows us to make the (para-)quaternionic structure manifest, and to show that the two reductions carry additional geometrical structures which are not preserved by the local isometry relating them.

To proceed, we introduce the following basis for the 1-forms on the scalar manifold:

$$
\begin{aligned}
\eta^{2} & =\frac{1}{\phi}\left(d \tilde{\phi}+p^{I} d s_{I}-s_{I} d p^{I}\right), \quad \xi_{2}=\frac{d \phi}{\phi}, \\
\alpha & =\frac{\sqrt{3}}{y} d x, \quad \beta=\frac{\sqrt{3}}{y} d y, \\
\eta^{0} & =\sqrt{\frac{y^{3}}{3 \phi}} d p^{0}, \quad \eta^{1}=\sqrt{\frac{y}{\phi}}\left(d p^{1}-x d p^{0}\right), \\
\xi_{0} & =2 \sqrt{\frac{3}{y^{3} \phi}}\left(d s_{0}+x d s_{1}+\frac{1}{2} x^{2} d p^{1}-\frac{1}{6} x^{3} d p^{0}\right), \\
\xi_{1} & =\frac{2}{\sqrt{y \phi}}\left(d s_{1}+x d p^{1}-\frac{1}{2} x^{2} d p^{0}\right) .
\end{aligned}
$$

These forms are also denoted

$$
\left(\theta^{a}\right)=\left(\eta^{2}, \xi_{2}, \alpha, \beta, \eta^{0}, \eta^{1}, \xi_{0}, \xi_{1}\right) .
$$

The metric $g$ on the target manifold associated with the Lagrangian (2.4) then takes the form

$$
4 g=-\epsilon_{1} \eta^{2} \otimes \eta^{2}+\xi_{2} \otimes \xi_{2}-\epsilon_{1} \alpha \otimes \alpha+\beta \otimes \beta-\epsilon \eta^{0} \otimes \eta^{0}-\epsilon_{2} \eta^{1} \otimes \eta^{1}-\epsilon_{2} \xi_{0} \otimes \xi_{0}-\epsilon \xi_{1} \otimes \xi_{1} .
$$

Note that under the analytic continuations (2.5) and (2.6) the one-forms (2.8) transform as

$$
\begin{aligned}
& \left(\eta^{2}, \xi_{2}, \alpha, \beta, \eta^{0}, \eta^{1}, \xi_{0}, \xi_{1}\right) \mapsto\left(i \eta^{2}, \xi_{2}, i \alpha, \beta,-i \eta^{0}, \eta^{1},-\xi_{0}, i \xi_{1}\right), \\
& \left(\eta^{2}, \xi_{2}, \alpha, \beta, \eta^{0}, \eta^{1}, \xi_{0}, \xi_{1}\right) \mapsto\left(-\eta^{2}, \xi_{2}, \alpha, \beta, i \eta^{0}, i \eta^{1}, i \xi_{0}, i \xi_{1}\right),
\end{aligned}
$$

which flips the relative signs in (2.9) in the same way as making the corresponding changes in the parameters $\epsilon_{1}$ and $\epsilon_{2}$. 
The one-forms $\theta^{a}$ have the following exterior derivatives:

$$
\begin{aligned}
d \eta^{2} & =-\xi_{0} \wedge \eta^{0}-\xi_{1} \wedge \eta^{1}-\xi_{2} \wedge \eta^{2}, \\
d \xi_{2} & =0 \\
d \alpha & =\frac{1}{\sqrt{3}} \alpha \wedge \beta, \\
d \beta & =0 \\
d \eta^{0} & =\frac{\sqrt{3}}{2} \beta \wedge \eta^{0}-\frac{1}{2} \xi_{2} \wedge \eta^{0}, \\
d \eta^{1} & =\frac{1}{2 \sqrt{3}} \beta \wedge \eta^{1}-\frac{1}{2} \xi_{2} \wedge \eta^{1}-\alpha \wedge \eta^{0}, \\
d \xi_{0} & =-\frac{\sqrt{3}}{2} \beta \wedge \xi_{0}-\frac{1}{2} \xi_{2} \wedge \xi_{0}+\alpha \wedge \xi_{1}, \\
d \xi_{1} & =-\frac{1}{2 \sqrt{3}} \beta \wedge \xi_{1}-\frac{1}{2} \xi_{2} \wedge \xi_{1}+\frac{2}{\sqrt{3}} \alpha \wedge \eta^{1} .
\end{aligned}
$$

This shows that they form a Lie algebra and that $g$ can be considered as a left-invariant pseudo-Riemannian metric on the corresponding simply connected Lie group, which is parametrized by $\left(x, y, \phi, \tilde{\phi}, p^{0}, p^{1}, s_{0}, s_{1}\right)$. The structure constants of this Lie algebra can be read off from the relation $d \theta^{c}=-c_{a b}^{c} \theta^{a} \wedge \theta^{b}$. The relations for the dual vector fields $T_{a}$, where $\left\langle\theta^{a}, T_{b}\right\rangle=\delta_{b}^{a}$, which we identify with the Lie algebra generators, are $\left[T_{a}, T_{b}\right]=c_{a b}^{c} T_{c}$.

Denoting the basis dual to $\left(\theta^{a}\right)$ by

$$
\left(T_{a}\right)=\left(V_{2}, U^{2}, A, B, V_{0}, V_{1}, U^{0}, U^{1}\right)
$$

we obtain:

$$
\begin{aligned}
{[B, A] } & =\frac{1}{\sqrt{3}} A, \quad\left[U^{2}, V_{2}\right]=V_{2}, \\
{\left[V_{0}, U^{0}\right] } & =-V_{2}, \quad\left[V_{1}, U^{1}\right]=-V_{2}, \\
{\left[U^{2}, V_{I}\right] } & =\frac{1}{2} V_{I} \quad \text { for } I=0,1, \quad\left[U^{2}, U^{I}\right]=\frac{1}{2} U^{I} \quad \text { for } I=0,1, \\
{\left[B, V_{0}\right] } & =-\frac{\sqrt{3}}{2} V_{0}, \quad\left[B, V_{1}\right]=-\frac{1}{2 \sqrt{3}} V_{1}, \quad\left[B, U^{0}\right]=\frac{\sqrt{3}}{2} U^{0}, \\
{\left[B, U^{1}\right] } & =\frac{1}{2 \sqrt{3}} U^{1}, \\
{\left[A, V_{0}\right] } & =V_{1}, \quad\left[A, U^{1}\right]=-U^{0}, \quad\left[A, V_{1}\right]=-\frac{2}{\sqrt{3}} U^{1} .
\end{aligned}
$$

This Lie algebra is easily seen to be a solvable Lie algebra. As we will see below, it is an Iwasawa subalgebra of the Lie algebra of $G_{2(2)}$. Thus the three dimensional reductions provide us with scalar manifolds which can all be identified with the group manifold $L$ of an Iwasawa subgroup of $G_{2(2)}$. For each of the three reductions this manifold is equipped with a different left-invariant metric. The signature is, using the ordering (2.8),

$$
\operatorname{sign}(g)=\left(-\epsilon_{1},+,-\epsilon_{1},+,-\epsilon,-\epsilon_{2},-\epsilon_{2},-\epsilon\right) .
$$


Thus for an SS reduction the metric is positive definite, while for ST and TS reductions we obtain split (i.e. neutral) signature metrics, but with a different distribution of $(+)$-signs and (-)-signs. Note that while scalar products are classified up to isomorphism by their signatures, this does not imply the existence of an isometry which simultaneously preserves the Lie algebra structure. This will be important in the following.

\section{The group $G_{2(2)}$, its Iwasawa subgroup, and the symmetric space $S=$ $G_{2(2)} /(\mathrm{SL}(2) \cdot \mathrm{SL}(2))$}

\subsection{The noncompact group of type G2}

Let us denote by $G=G_{2(2)}$ the simply connected noncompact form of the simple Lie group of type G2. Its Lie algebra $\mathfrak{g}$ can be described as follows, see [30], Ch. 5, section 1.2. It contains $\mathfrak{s l}(V)$ as a subalgebra, where $V=\mathbb{R}^{3}$, such that under the adjoint representation of $\mathfrak{s} l(V)$ on $\mathfrak{g}$ we have the following decomposition

$$
\mathfrak{g}=V+\mathfrak{s l}(V)+V^{*}
$$

as a direct sum of irreducible $\mathfrak{s l}(V)$-submodules. The remaining Lie brackets are given by

$$
\begin{aligned}
& {[x, y]=-2 x \times y,} \\
& {[\xi, \eta]=2 \xi \times \eta} \\
& {[x, \xi]=3 x \otimes \xi-\xi(x) \operatorname{Id} \in \mathfrak{s l}(V) \subset \mathfrak{g l}(V) \cong V \otimes V^{*},}
\end{aligned}
$$

for all $x, y \in V, \xi, \eta \in V^{*}$. The cross products are defined by

$$
x \times y=\operatorname{det}(x, y, \cdot) \in V^{*}, \quad \xi \times \eta=\operatorname{det}^{-1}(\xi, \eta, \cdot) \in V^{* *}=V,
$$

where $\operatorname{det}^{-1} \in \wedge^{3} V$ is the inverse of $\operatorname{det} \in \wedge^{3} V^{*}$. Let us denote by $\mathfrak{a}$ the Cartan subalgebra of $\mathfrak{g}$ which consists of all diagonal matrices in $\mathfrak{s l}(V)$. We shall denote by $\left(e_{i}\right)=\left(e_{1}, e_{2}, e_{3}\right)$ the standard basis of $V$, by $\left(e^{i}\right)$ its dual basis and by $e_{i}^{j}$ the endomorphism $e_{i} \otimes e^{j}$ of $V$. With this notation,

$$
\mathfrak{a}=\left\{\sum \lambda_{i} e_{i}^{i} \mid \sum \lambda_{i}=0\right\}
$$

3.2 The symmetric para-quaternionic-Kähler manifold $S=G_{2(2)} /\left(\mathrm{SL}_{2} \cdot \mathrm{SL}_{2}\right)$

Proposition 1 The Lie algebra $\mathfrak{g}$ admits the following $\mathbb{Z}_{2}$-grading

$$
\mathfrak{g}=\mathfrak{g}_{e v}+\mathfrak{g}_{\text {odd }}
$$

where

$$
\begin{aligned}
\mathfrak{g}_{e v} & =\mathfrak{a}+\operatorname{span}\left\{e_{3}, e^{3}, e_{1}^{2}, e_{2}^{1}\right\} \cong \mathfrak{s l}_{2} \oplus \mathfrak{s l}_{2}, \\
\mathfrak{g}_{\text {odd }} & =\operatorname{span}\left\{e_{1}, e_{2}, e^{1}, e^{2}, e_{1}^{3}, e_{2}^{3}, e_{3}^{1}, e_{3}^{2}\right\} .
\end{aligned}
$$

The corresponding symmetric space $S=G / G_{e v}$ admits a $G$-invariant para-quaternionicKähler structure $(g, Q)$, unique up to scale. The metric $g$ is induced by a multiple of the Killing form. 
Proof: It is straightforward to check that (3.1) is a $\mathbb{Z}_{2}$-grading of the Lie algebra $\mathfrak{g}$. This shows that $S$ is a symmetric space. Furthermore,

$$
\left(\mathbf{h}=\left[e_{1}^{2}, e_{2}^{1}\right]=e_{1}^{1}-e_{2}^{2}, \mathbf{e}=e_{1}^{2}, \mathbf{f}=e_{2}^{1}\right),
$$

is an $\mathfrak{s l}_{2}$-triple $(\mathbf{h}, \mathbf{e}, \mathbf{f})$, as well as

$$
\left(\left[e_{3}, e^{3}\right]=-e_{1}^{1}-e_{2}^{2}+2 e_{3}^{3}, e_{3}, e^{3}\right) .
$$

They generate two complementary ideals $\mathfrak{s l}_{2}^{(a)} \cong \mathfrak{s l}_{2}, a=1,2$, in $\mathfrak{g}_{e v}$. One can further check that the isotropy representation of $S$ is a tensor product $\mathbb{R}^{2} \otimes \mathbb{R}^{4}$ of irreducible representations of the two $S L_{2}$-factors. The total irreducibility of the isotropy representation implies that the metric induced by the Killing form is the only $G$-invariant pseudo-Riemannian metric $g$ on $S$, up to scale. The isotropy representation of $\mathfrak{s l}_{2}^{(1)} \subset \mathfrak{g}_{e v}$ on the first factor defines a $G$-invariant almost para-quaternionic structure $Q$ on $S$, which consists of skewsymmetric endomorphisms. It is the only $G$-invariant almost para-quaternionic structure on $S$, since the $G_{e v}$-invariant decomposition $T_{o} S \cong \mathbb{R}^{2} \otimes \mathbb{R}^{4}$ is unique, where $o=e G_{e v}$ stands for the canonical base point of $S=G / G_{e v}$. Moreover, $Q$ is invariant under parallel transport because the isotropy group coincides with the holonomy group of the simply connected pseudo-Riemannian symmetric space $S$, as a consequence of the Ambrose-Singer theorem. So $(S, g, Q)$ is a para-quaternionic-Kähler manifold.

\subsection{The solvable Iwasawa subgroup $L \subset G$}

Let us define

$$
\mathfrak{n}:=\operatorname{span}\left\{e_{1}, e^{2}, e^{3}, e_{1}^{2}, e_{1}^{3}, e_{2}^{3}\right\} \subset \mathfrak{g} .
$$

We claim that $\mathfrak{n} \subset \mathfrak{g}$ is a maximal unipotent ${ }^{2}$ subalgebra normalized by the Cartan subalgebra $\mathfrak{a} \subset \mathfrak{g}$. In fact, $\mathfrak{n}$ is precisely the sum of the positive root spaces of $\mathfrak{a}$ with respect to the Weyl chamber containing the element $3 e_{1}^{1}-e_{2}^{2}-2 e_{3}^{3} \in \mathfrak{a}$. As a consequence, we obtain:

Proposition 2 The solvable Lie algebra

$$
\mathfrak{l}=\mathfrak{a}+\mathfrak{n} \subset \mathfrak{g}
$$

is a maximal triangular subalgebra of $\mathfrak{g}$.

Any maximal triangular subalgebra of $\mathfrak{g}$ will be called an Iwasawa subalgebra, since it is the solvable Lie algebra appearing in the Iwasawa decomposition of $\mathfrak{g}$. Any two Iwasawa subalgebras of $\mathfrak{g}$ are conjugated.

\footnotetext{
${ }^{2} \mathrm{~A}$ subalgebra of a linear Lie algebra is called unipotent if it operates on the given vector space by upper triangular matrices with vanishing diagonal elements. Note that a nilpotent Lie algebra is not automatically unipotent if the Lie algebra is represented by matrices. For the adjoint representation it is true that nilpotent Lie algebras are realized as unipotent linear Lie algebras, but this is not necessarily true for other representations. Since the representation we use is not the adjoint representation of $\mathfrak{n}$, but the restriction of the adjoint representation of $\mathfrak{g}$ to $\mathfrak{n}$, the distinction between nilpotent and unipotent subalgebras is relevant.
} 
The Iwasawa decomposition implies that the Lie subgroup $L \subset G$ with the Lie algebra $\mathfrak{l} \subset \mathfrak{g}$ acts simply transitively on the quaternionic-Kähler symmetric space $G / S O_{4}$. Therefore, the quaternionic-Kähler structure can be described as a left-invariant structure on $L$. This was done in [10]. Correcting some misprints and changing slightly the notation, the Lie algebra of the simply transitive group described by Alekseevsky is spanned by a basis

$$
\left(G_{0}, H_{0}, G_{1}, H_{1}, \tilde{P}_{-}, \tilde{P}_{+}, \tilde{Q}_{-}, \tilde{Q}_{+}\right)
$$

with the following nontrivial brackets:

$$
\begin{aligned}
{\left[H_{0}, G_{0}\right] } & =G_{0},\left[H_{1}, G_{1}\right]=\frac{1}{\sqrt{3}} G_{1}, \\
{\left[H_{0}, \tilde{U}\right] } & =\frac{1}{2} \tilde{U}, \quad \text { for all } \quad \tilde{U} \in \tilde{\mathfrak{u}}:=\operatorname{span}\left\{\tilde{P}_{-}, \tilde{P}_{+}, \tilde{Q}_{-}, \tilde{Q}_{+}\right\} \\
{\left[H_{1}, \tilde{P}_{-}\right] } & =\frac{\sqrt{3}}{2} \tilde{P}_{-},\left[H_{1}, \tilde{P}_{+}\right]=\frac{1}{2 \sqrt{3}} \tilde{P}_{+},\left[H_{1}, \tilde{Q}_{-}\right]=-\frac{\sqrt{3}}{2} \tilde{Q}_{-},\left[H_{1}, \tilde{Q}_{+}\right]=-\frac{1}{2 \sqrt{3}} \tilde{Q}_{+}, \\
{\left[G_{1}, \tilde{P}_{+}\right] } & =-\tilde{P}_{-},\left[G_{1}, \tilde{Q}_{-}\right]=\tilde{Q}_{+},\left[G_{1}, \tilde{Q}_{+}\right]=\frac{2}{\sqrt{3}} \tilde{P}_{+}, \\
{\left[\tilde{Q}_{-}, \tilde{P}_{-}\right] } & =\left[\tilde{Q}_{+}, \tilde{P}_{+}\right]=G_{0} .
\end{aligned}
$$

Proposition 3 The Lie algebra $\mathfrak{l}$ admits a basis $\left(G_{0}, H_{0}, G_{1}, H_{1}, \tilde{P}_{-}, \tilde{P}_{+}, \tilde{Q}_{-}, \tilde{Q}_{+}\right)$with the above commutators.

Proof: It suffices to define

$$
\begin{aligned}
& G_{0}:=-3 e_{1}^{3}, H_{0}:=\frac{1}{2}\left(e_{1}^{1}-e_{3}^{3}\right), G_{1}:=\frac{1}{\sqrt{3}} e^{2}, H_{1}:=\frac{1}{2 \sqrt{3}}\left(e_{1}^{1}-2 e_{2}^{2}+e_{3}^{3}\right) \\
& \tilde{P}_{-}:=\sqrt{3} e_{1}^{2}, \tilde{P}_{+}:=e_{1}, \tilde{Q}_{-}:=\sqrt{3} e_{2}^{3}, \tilde{Q}_{+}:=e^{3} .
\end{aligned}
$$

To compare with the results obtained by dimensional reduction it is more convenient to work with the following basis:

$$
\left(\mathcal{V}_{1}, \ldots, \mathcal{V}_{8}\right)=\left(G_{0}, H_{0}, G_{1}, H_{1}, \tilde{Q}_{-}, \tilde{Q}_{+},-\tilde{P}_{-},-\tilde{P}_{+},\right)
$$

i.e.

$$
\begin{aligned}
& \mathcal{V}_{1}=-3 e_{1}^{3}, \mathcal{V}_{2}=\frac{1}{2}\left(e_{1}^{1}-e_{3}^{3}\right), \mathcal{V}_{3}=\frac{1}{\sqrt{3}} e^{2}, \mathcal{V}_{4}=\frac{1}{2 \sqrt{3}}\left(e_{1}^{1}-2 e_{2}^{2}+e_{3}^{3}\right), \\
& \mathcal{V}_{5}=\sqrt{3} e_{2}^{3}, \nu_{6}=e^{3}, \mathcal{V}_{7}:=-\sqrt{3} e_{1}^{2}, \mathcal{V}_{8}:=-e_{1}
\end{aligned}
$$

which has precisely the same nontrivial brackets (2.12) as the basis $T_{a}$ of the Lie algebra obtained from dimensional reduction. 


\section{Realization of the scalar manifolds of the reduced theories as open orbits in the symmetric space $S$}

Our goal is to realize the scalar manifolds $M^{(T S)}$ and $M^{(S T)}$ of the reduced theories as open orbits $M_{1}=L^{\prime} \cdot o$ and $M_{2}=L^{\prime \prime} \cdot o$ of Iwasawa subgroups $L^{\prime}, L^{\prime \prime} \subset G$ on the symmetric space $S=G / G_{e v}$. Notice that the standard Iwasawa subgroup $L \subset G$ acts transitively on the Riemannian symmetric space $G / S_{4}$, but that the orbit $L \cdot o$ of the canonical base point $o \in G /(\mathrm{SL}(2) \cdot \mathrm{SL}(2))$ under this group is not even open. Our strategy is to look for a conjugate subgroup $L^{\prime}=C_{a}(L)=a L a^{-1}, a \in G$, such that the orbit $M_{1}=L^{\prime} \cdot o$ is open, and then to try to show that $M_{1}$ is isometrically covered, up to a positive scale factor, by at least one of the two scalar manifolds $M^{(T S)}$ or $M^{(S T)}$.

In the following subsection we construct an Iwasawa subgroup $L^{\prime}=C_{a}(L) \subset G$ for which the orbit $L^{\prime} \cdot o \subset S$ is open. Composing the isomorphism $C_{a}: L \rightarrow L^{\prime}$ defined by conjugation by $a \in G$ with the covering $L^{\prime} \rightarrow M_{1}$ given by the orbit map $x \mapsto x \cdot o$ we obtain a $C_{a}$-equivariant covering $\phi_{1}: L \rightarrow M_{1}$ and a left-invariant metric $g_{1}=\phi_{1}^{*} g_{S}$ on $L$, which we can compare to the metrics $g^{(T S)}=g^{(1,-1)}$ and $g^{(S T)}=g^{(-1,1)}$. Recall that the solvable Lie algebra obtained from dimensional reduction comes equipped with the basis $\mathcal{T}:=\left(T_{1}, \ldots, T_{8}\right)$, whereas the standard Iwasawa Lie subalgebra $\mathfrak{l} \subset \mathfrak{g}$ is equipped with the basis $\mathcal{V}:=\left(\mathcal{V}_{1}, \ldots, \mathcal{V}_{8}\right)$. Since these bases have the same structure constants, we can identify the two Lie algebras. In Proposition 5 we compute the Gram matrix $\mathcal{G}_{1}$ of $g_{1}$ with respect to the basis $\mathcal{V}$. Contrary to the metrics $g^{(T S)}$ and $g^{(S T)}$, for which the basis $\mathcal{T}$ is orthonormal (up to an overall factor), we find that $\mathcal{G}_{1}$ is not even diagonal. Notice that nevertheless the left-invariant metrics $g_{1}$ and $g^{(T S)}$ or $g^{(S T)}$ on $L$ could be equivalent, that is related by an automorphism of $L$, up to a positive scale factor. In subsection 4.2 we determine the group $\operatorname{Aut}(L)$ of all automorphisms of $L$. As a result we find in particular that for the connected component of the identity $\operatorname{Aut}_{0}(L)=\operatorname{Inn}(L) \cong L$. In subsection 4.3 we prove that the metrics $g_{1}$ and $g^{(T S)}$ are equivalent and, more precisely, related by a unique inner automorphism of $L$, and multiplication by a factor of 2 . Similarly in subsection 4.4 we construct a second Iwasawa subgroup $L^{\prime \prime} \subset G$ such that $M_{2}=L^{\prime \prime} \cdot o$ is open and a covering $\phi_{2}: L \rightarrow M_{2}$ which is equivariant with respect to an isomorphism $L \rightarrow L^{\prime \prime}$. Finally, the left-invariant metric $g_{2}=\phi_{2}^{*} g_{S}$ is shown to be related to the metric $g^{(S T)}$ by a unique inner automorphism of $L$, and multiplication by a factor of 2 . We also show that, surprisingly, the metrics $g_{1}$ and $g_{2}$ are not related by any automorphism. We will see in section 5 that the metric Lie groups $\left(L, g_{1}\right)$ and $\left(L, g_{2}\right)$ have different geometric properties.

\subsection{Iwasawa subgroups of $G$ with an open orbit on $S$}

Given a subgroup $U \subset G$ we can consider the orbit $U \cdot o \subset S=G / G_{e v}$ of the canonical base point $o \in S$. The orbit is open if and only if $\mathfrak{g}_{e v}+\operatorname{Lie}(U)=\mathfrak{g}$. For an Iwasawa subalgebra $\mathfrak{l}^{\prime}=\operatorname{Ad}_{a} \mathfrak{l} \subset \mathfrak{g}, a \in G$, this is the case if and only if $\mathfrak{g}_{e v} \cap \mathfrak{l}^{\prime}=0$. In that case, the orbit map $L^{\prime} \rightarrow M=L^{\prime} \cdot o \subset S$ is a covering and we obtain a left-invariant locally symmetric para-quaternionic-Kähler structure on $L^{\prime} \cong L$ induced from the symmetric para- 
quaternionic-Kähler structure on $S$. Notice that the orbit $L \cdot o$ (the case $a=e$ ) is not open, since $\mathfrak{g}_{e v} \cap \mathfrak{l} \neq 0$.

Proposition 4 The element $a=\exp \xi$, where $\xi=e^{1}+e_{3}^{1} \in \mathfrak{g}$, defines an Iwasawa subalgebra $\mathfrak{l}^{\prime}=\operatorname{Ad}_{a} \mathfrak{l} \subset \mathfrak{g}$ transversal to $\mathfrak{g}_{e v}$.

Proof: We first compute $X^{\prime}:=\operatorname{Ad}_{a} X=e^{a d_{\xi}} X$ for every element $X \in \mathfrak{l}$. For $H=\sum \lambda_{i} e_{i}^{i} \in$ $\mathfrak{a} \subset \mathfrak{l}$,

$$
a d_{\xi} H=-[H, \xi]=-\left(-\lambda_{1} e^{1}+\left(-\lambda_{1}+\lambda_{3}\right) e_{3}^{1}\right)=\lambda_{1} e^{1}+\left(\lambda_{1}-\lambda_{3}\right) e_{3}^{1}, a d_{\xi}^{2} H=0,
$$

implies

$$
H^{\prime}=H+\lambda_{1} e^{1}+\left(\lambda_{1}-\lambda_{3}\right) e_{3}^{1}
$$

Next,

$$
\begin{aligned}
a d_{\xi} e_{1} & =-2 e_{1}^{1}+e_{2}^{2}+e_{3}^{3}+e_{3}, \\
a d_{\xi}^{2} e_{1} & =-2\left[e^{1}, e_{1}^{1}\right]+\left[e^{1}, e_{3}\right]-2\left[e_{3}^{1}, e_{1}^{1}\right]+\left[e_{3}^{1}, e_{3}^{3}\right] \\
& =-2 e^{1}-3 e_{3}^{1}-2 e_{3}^{1}-e_{3}^{1}=-2\left(e^{1}+3 e_{3}^{1}\right), \\
a d_{\xi}^{3} e_{1} & =0,
\end{aligned}
$$

implies

$$
\begin{aligned}
e_{1}^{\prime} & =e_{1}-2 e_{1}^{1}+e_{2}^{2}+e_{3}^{3}+e_{3}-e^{1}-3 e_{3}^{1} . \\
a d_{\xi} e^{2} & =2 e_{3}, a d_{\xi}^{2} e^{2}=-6 e_{3}^{1}, a d_{\xi}^{3} e^{2}=0 \Longrightarrow \\
e^{2^{\prime}} & =e^{2}+2 e_{3}-3 e_{3}^{1} . \\
a d_{\xi} e^{3} & =-2 e_{2}-e^{1}, a d_{\xi}^{2} e^{3}=6 e_{2}^{1}, a d_{\xi}^{3} e^{3}=0 \Longrightarrow \\
e^{3^{\prime}} & =e^{3}-2 e_{2}-e^{1}+3 e_{2}^{1} . \\
a d_{\xi} e_{1}^{2} & =e^{2}+e_{3}^{2}, a d_{\xi}^{2} e_{1}^{2}=2 e_{3}, a d_{\xi}^{3} e_{1}^{2}=-6 e_{3}^{1}, a d_{\xi}^{4} e_{1}^{2}=0 \Longrightarrow \\
e_{1}^{2^{\prime}} & =e_{1}^{2}+e^{2}+e_{3}^{2}+e_{3}-e_{3}^{1} . \\
a d_{\xi} e_{1}^{3} & =e^{3}+e_{3}^{3}-e_{1}^{1}, a d_{\xi}^{2} e_{1}^{3}=-2\left(e_{2}+e^{1}+e_{3}^{1}\right), a d_{\xi}^{3} e_{1}^{3}=6 e_{2}^{1}, a d_{\xi}^{4} e_{1}^{3}=0 \Longrightarrow \\
e_{1}^{3^{\prime}} & =e_{1}^{3}+e^{3}+e_{3}^{3}-e_{1}^{1}-e_{2}-e^{1}-e_{3}^{1}+e_{2}^{1} . \\
a d_{\xi} e_{2}^{3} & =-e_{2}^{1}, a d_{\xi}^{2} e_{1}^{3}=0 \Longrightarrow \\
e_{2}^{3^{\prime}} & =e_{2}^{3}-e_{2}^{1} .
\end{aligned}
$$

Next we check the transversality of $\mathfrak{l}^{\prime}$. Let us denote by $\pi: \mathfrak{g} \rightarrow \mathfrak{g}_{\text {odd }}$ the projection along $\mathfrak{g}_{e v}$ and by $\varphi: \mathfrak{l} \rightarrow \mathfrak{g}_{\text {odd }}$ the map $X \mapsto \pi\left(X^{\prime}\right)$. From (4.1)-(4.7) we can read off $\varphi$ :

$$
\begin{aligned}
& \varphi(H)=\lambda_{1} e^{1}+\left(\lambda_{1}-\lambda_{3}\right) e_{3}^{1}, \quad \text { for all } H=\sum \lambda_{i} e_{i}^{i} \in \mathfrak{a}, \\
& \varphi\left(e_{1}\right)=e_{1}-e^{1}-3 e_{3}^{1}, \varphi\left(e^{2}\right)=e^{2}-3 e_{3}^{1}, \varphi\left(e^{3}\right)=-2 e_{2}-e^{1}, \\
& \varphi\left(e_{1}^{2}\right)=e^{2}+e_{3}^{2}-e_{3}^{1}, \varphi\left(e_{1}^{3}\right)=e_{1}^{3}-e_{2}-e^{1}-e_{3}^{1}, \varphi\left(e_{2}^{3}\right)=e_{2}^{3},
\end{aligned}
$$

which shows that $\varphi: \mathfrak{l} \rightarrow \mathfrak{g}_{\text {odd }}$ is an isomorphism of vector spaces. This implies that $\mathfrak{l}^{\prime}$ is transversal to $\mathfrak{g}_{e v}$. 
Next we compute the left-invariant metric $g_{1}$ on $L \cong L^{\prime}$ which corresponds to the locally symmetric para-quaternionic-Kähler manifold $M_{1}=L^{\prime} \cdot o \subset S$. Let us denote by $B$ the Killing form of $\mathfrak{g}$ and by $\langle\cdot, \cdot\rangle_{B}$ the scalar product on $\mathfrak{g}_{\text {odd }}$ obtained by restricting $\frac{1}{8} B$.

Lemma 1 The nontrivial scalar products between elements of the basis $\left(e_{1}, e_{2}, e^{1}, e^{2}, e_{1}^{3}, e_{2}^{3}, e_{3}^{1}, e_{3}^{2}\right)$ of $\mathfrak{g}_{\text {odd }}$ are precisely:

$$
\left\langle e^{1}, e_{1}\right\rangle_{B}=\left\langle e^{2}, e_{2}\right\rangle_{B}=3,\left\langle e_{2}^{3}, e_{3}^{2}\right\rangle_{B}=\left\langle e_{1}^{3}, e_{3}^{1}\right\rangle_{B}=1 .
$$

The scalar product $\langle\cdot, \cdot\rangle_{1}$ on $\mathfrak{l}$ which defines the metric $g_{1}$ is precisely the pull back of $\langle\cdot, \cdot\rangle_{B}$ by the isomorphism $\varphi=\pi \circ \operatorname{Ad}_{a}: \mathfrak{l} \rightarrow \mathfrak{g}_{\text {odd }}$.

Proposition 5 The matrix representing the scalar product $\langle\cdot, \cdot\rangle_{1}=\varphi^{*}\langle\cdot, \cdot\rangle_{B}$ in the basis V is:

$$
\mathcal{G}_{1}=\left(\begin{array}{rrrrrrrr}
-18 & -3 & 6 \sqrt{3} & 0 & 0 & 0 & -12 \sqrt{3} & -18 \\
-3 & 0 & 0 & 0 & 0 & 0 & 0 & -\frac{3}{2} \\
6 \sqrt{3} & 0 & 0 & 0 & 0 & -2 \sqrt{3} & 0 & 0 \\
0 & 0 & 0 & 0 & 0 & 0 & 0 & -\frac{\sqrt{3}}{2} \\
0 & 0 & 0 & 0 & 0 & 0 & -3 & 0 \\
0 & 0 & -2 \sqrt{3} & 0 & 0 & 0 & 6 \sqrt{3} & 3 \\
-12 \sqrt{3} & 0 & 0 & 0 & -3 & 6 \sqrt{3} & 0 & 0 \\
-18 & -\frac{3}{2} & 0 & -\frac{\sqrt{3}}{2} & 0 & 3 & 0 & -6
\end{array}\right) .
$$

Proof: This follows from (4.8) with the help of Lemma 1

To compare the above left-invariant metric $g_{1}$ with the metrics obtained from dimensional reduction we need to study the automorphism group of the solvable Lie group $L$. Since $L$ is simply connected, we have $\operatorname{Aut}(L) \cong \operatorname{Aut}(\mathfrak{l})$.

\subsection{Automorphisms of the solvable algebra}

In this subsection we determine the automorphism group of the solvable Lie algebra $\mathfrak{l}$. For the proof we will use the following dual characterization of automorphisms.

Proposition 6 Given a Lie algebra $\mathfrak{l}$, an invertible linear map $\Lambda: \mathfrak{l} \rightarrow \mathfrak{l}$ is an automorphism if and only if

$$
d \Lambda^{*} \theta=\Lambda^{*} d \theta
$$

for all $\theta \in \mathfrak{l}^{*}$.

Recall that given a basis $\left(T_{a}\right)$ of a Lie algebra $\mathfrak{l}$ with structure constants $c_{a b}^{c}$, that is $\left[T_{a}, T_{b}\right]=c_{a b}^{c} T_{c}$, the differential is given in terms of the dual basis $\left(\theta^{a}\right)$ as follows

$$
d \theta^{a}=-c_{b c}^{a} \theta^{b} \wedge \theta^{c} .
$$

In other words, $\Lambda$ is an automorphism if and only if the dual map $\Omega=\Lambda^{*}$ satisfies

$$
d \Omega\left(\theta^{a}\right)=-c_{b c}^{a} \Omega\left(\theta^{b}\right) \wedge \Omega\left(\theta^{c}\right)
$$

for all $a=1, \ldots, \operatorname{dim}(\mathfrak{l})$. 
For the Iwasawa Lie algebra $\mathfrak{l}$ we can use the basis $\left(T_{a}\right)=\left(\mathcal{V}_{a}\right)$ defined in (2.11). The differentials of the dual basis $\left(\theta^{a}\right)$ are given by $(2.10)$, if we put $\left(\eta^{2}, \xi_{2}, \alpha, \beta, \eta^{0}, \eta^{1}, \xi_{0}, \xi_{1}\right)=$ $\left(\theta^{1}, \ldots, \theta^{8}\right)$.

We now show the following:

Theorem 1 The group of automorphisms of the solvable Lie algebra $\mathfrak{l}$ is given by Aut $(\mathfrak{l})=$ $\left(\mathbb{Z}_{2} \times \mathbb{Z}_{2}\right) \ltimes \operatorname{Inn}(\mathfrak{l})$, where $\operatorname{Inn}(\mathfrak{l}) \cong L$ denotes the group of inner automorphisms of $\mathfrak{l}$, and the generators of the cyclic factors of the group

$\mathbb{Z}_{2} \times \mathbb{Z}_{2} \subset \operatorname{Aut}(\mathfrak{l})$ act by the diagonal matrices

$$
\operatorname{diag}(-1,1,-1,1,1,-1,-1,1) \quad \text { and } \operatorname{diag}(1,1,1,1,-1,-1,-1,-1) \text {, }
$$

on the Lie algebra $\mathfrak{l}$ with respect to the basis (2.11). An explicit parametrization of the group $\operatorname{Aut}(\mathfrak{l})$ by

$$
\left\{(a, b, c, d, e, f, g, h) \in \mathbb{R}^{8} \mid b e \neq 0\right\} \cong \mathbb{R}^{*} \times \mathbb{R}^{*} \times \mathbb{R}^{6},
$$

is given by the matrix (4.12), which represents the action of the group element with parameters $(a, b, c, d, e, f, g, h)$ in the given basis of $\mathfrak{l}$.

Proof: We work with the 1-forms (2.8), which have exterior derivatives (2.10).

We first note that the six non-zero differentials which appear on the right-hand side of (2.10) are linearly independent. Hence, the space of closed one-forms $Z^{1}(\mathfrak{l})$ is spanned by $\left\{\xi_{2}, \beta\right\}$.

In order to determine all automorphisms $\Lambda$ of $\mathfrak{l}$ we consider $\Omega=\Lambda^{*}$ and define coefficients $\Omega^{a}{ }_{b}$ by $\Omega\left(\theta^{a}\right)=\Omega^{a}{ }_{b} \theta^{b}$, such that $\mathcal{M}=\left(\mathcal{M}_{a}^{b}\right)_{a, b}=\left(\Omega^{b}{ }_{a}\right)_{a, b}$ is the matrix representing $\Omega$ with respect to the basis $\left(\theta^{a}\right)$, and, hence, is the transpose of the matrix representing $\Lambda$ with respect to the basis $\left(T_{a}\right)$. We then simply work through each of the basis 1-forms $\left(\theta^{a}\right)$ and determine the coefficients $\Omega^{a}{ }_{b}$ such that (4.11) is satisfied. It turns out to be easiest to do this in the order $\xi_{2}, \beta, \alpha, \eta^{0}, \eta^{1}, \xi_{1}, \xi_{0}, \eta^{2}$.

Since any automorphism preserves $Z^{1}(\mathfrak{l})=\operatorname{span}\left\{\xi_{2}, \beta\right\}$ we see that

$$
\Omega\left(\xi_{2}\right)=\Omega^{2}{ }_{2} \xi_{2}+\Omega^{2}{ }_{4} \beta, \quad \Omega(\beta)=\Omega^{4}{ }_{2} \xi_{2}+\Omega^{4}{ }_{4} \beta .
$$

We now turn to the 1 -form $\alpha$. The automorphism $\Omega$ must satisfy

$$
d \Omega(\alpha)=\frac{1}{\sqrt{3}} \Omega(\alpha) \wedge \Omega(\beta) .
$$

Since we have already determined that $\Omega(\beta)$ should be a linear combination of $\left\{\xi_{2}, \beta\right\}$, we deduce that $\Omega(\alpha)$ should only contain terms whose exterior derivative has a $\xi_{2}$ or $\beta$ in every term. Hence, we require

$$
\Omega(\alpha)=\Omega^{3}{ }_{2} \xi_{2}+\Omega^{3}{ }_{3} \alpha+\Omega^{3}{ }_{4} \beta+\Omega^{3}{ }_{5} \eta^{0} .
$$

We next use the automorphism condition to find algebraic relations between the components of $\Omega^{3}{ }_{a}$ and $\Omega^{4}{ }_{a}$. In particular, we have

$$
\Omega^{3}{ }_{2} \Omega^{4}{ }_{4}=\Omega^{3}{ }_{4} \Omega^{4}{ }_{2}, \quad \Omega^{3}{ }_{3}\left(\Omega^{4}{ }_{4}-1\right)=0, \quad \Omega^{3}{ }_{3} \Omega^{4}{ }_{2}=0,
$$




$$
\Omega^{3}{ }_{5}\left(\Omega^{4}{ }_{2}-\frac{\sqrt{3}}{2}\right)=0, \quad \Omega^{3}{ }_{5}\left(\Omega^{4}{ }_{4}+\frac{3}{2}\right)=0 .
$$

From this we see that we can't have both $\Omega^{3}{ }_{3}$ and $\Omega^{3}{ }_{5}$ being non-zero. We consider the case $\Omega^{3}{ }_{3}:=b \neq 0, \Omega^{3}{ }_{5}=0$, which restricts

$$
\Omega_{2}^{4}=0, \quad \Omega_{4}^{4}=1, \quad \Omega^{3}{ }_{2}=0 .
$$

It turns out that the other possible choice $\Omega^{3}{ }_{3}=0, \Omega^{3}{ }_{5} \neq 0$ does not give rise to an invertible linear map $\Omega$. The corresponding analysis is omitted.

By successively analysing all algebraic relations, we find the most general automorphism of $\mathfrak{l}$, which depends on eight real parameters

$$
\Omega^{1}{ }_{2}:=a, \Omega^{3}{ }_{3}:=b, \Omega^{3}{ }_{4}:=c, \Omega^{5}{ }_{2}:=d, \Omega_{5}^{5}:=e, \Omega^{6}{ }_{2}:=f, \Omega^{7}{ }_{2}:=g, \Omega^{8}{ }_{2}:=h,
$$

and is given by its action on the basis of 1 -forms $\left(\theta^{a}\right)$ as

$$
\begin{aligned}
\Omega\left(\xi_{2}\right)= & \xi_{2} \\
\Omega(\beta)= & \beta \\
\Omega(\alpha)= & b \alpha+c \beta \\
\Omega\left(\eta^{0}\right)= & d \xi_{2}-\sqrt{3} d \beta+e \eta^{0}, \\
\Omega\left(\eta^{1}\right)= & f \xi_{2}+2 b d \alpha+\left(2 c d-\frac{1}{\sqrt{3}} f\right) \beta-\sqrt{3} c e \eta^{0} \\
& +b e \eta^{1}, \\
\Omega\left(\xi_{1}\right)= & h \xi_{2}-\frac{4}{\sqrt{3}} b f \alpha+\left(\frac{1}{\sqrt{3}} h-\frac{4}{\sqrt{3}} c f\right) \beta-\sqrt{3} c^{2} e \eta^{0} \\
& +2 b c e \eta^{1}+b^{2} e \xi_{1}, \\
\Omega\left(\xi_{0}\right)= & g \xi_{2}-2 b h \alpha+(\sqrt{3} g-2 c h) \beta-c^{3} e \eta^{0} \\
& +\sqrt{3} b c^{2} e \eta^{1}+b^{3} e \xi_{0}+\sqrt{3} b^{2} c e \xi_{1}, \\
\Omega\left(\eta^{2}\right)= & b^{3} e^{2} \eta^{2}+a \xi_{2}-\left(4 b d h+\frac{4}{\sqrt{3}} b f^{2}\right) \alpha \\
& +\left(2 \sqrt{3} d g-4 c d h+\frac{2}{\sqrt{3}} f h-\frac{4}{\sqrt{3}} c f^{2}\right) \beta \\
& +\left(2 \sqrt{3} c e h-2 \sqrt{3} c^{2} e f-2 c^{3} d e-2 e g\right) \eta^{0} \\
& +\left(2 \sqrt{3} b c^{2} d e+4 b c e f-2 b e h\right) \eta^{1} \\
& +2 b^{3} d e \xi_{0}+\left(2 \sqrt{3} b^{2} c d e+2 b^{2} e f\right) \xi_{1} .
\end{aligned}
$$


This eight-parameter family describes all automorphisms of the Lie algebra $\mathfrak{l}$. We can now read off the matrix $\mathcal{M}$ representing $\Omega=\Lambda^{*}$ with respect to the basis $\left(\theta^{a}\right)$.

$$
\mathcal{M}=\left(\begin{array}{cccccccc}
b^{3} e^{2} & 0 & 0 & 0 & 0 & 0 & 0 & 0 \\
a & 1 & 0 & 0 & d & f & g & h \\
m_{3,1} & 0 & b & 0 & 0 & 2 b d & -2 b h & -4 / 3 \sqrt{3} b f \\
m_{4,1} & 0 & c & 1 & -\sqrt{3} d & m_{4,5} & \sqrt{3} g-2 c h & 1 / 3 \sqrt{3}(h-4 c f) \\
m_{5,1} & 0 & 0 & 0 & e & -\sqrt{3} c e & -c^{3} e & -\sqrt{3} c^{2} e \\
m_{6,1} & 0 & 0 & 0 & 0 & b e & \sqrt{3} b c^{2} e & 2 b c e \\
2 b^{3} d e & 0 & 0 & 0 & 0 & 0 & b^{3} e & 0 \\
m_{8,1} & 0 & 0 & 0 & 0 & 0 & \sqrt{3} b^{2} c e & b^{2} e
\end{array}\right),
$$

where

$$
\begin{array}{lll}
m_{3,1}=-4 b\left(d h+1 / 3 \sqrt{3} f^{2}\right), & m_{4,1}=2 \sqrt{3} d g-4 c d h+2 / 3 \sqrt{3} f h-4 / 3 \sqrt{3} c f^{2}, \\
m_{4,5}=2 c d-1 / 3 \sqrt{3} f, & m_{5,1}=2 \sqrt{3} c e h-2 \sqrt{3} c^{2} e f-2 c^{3} d e-2 e g, \\
m_{6,1}=2 b\left(\sqrt{3} c^{2} d e+2 c e f-e h\right), & m_{8,1}=2 b^{2}(\sqrt{3} c d e+e f) .
\end{array}
$$

Note that the matrix $\mathcal{M}$ satisfies the equation $\Lambda\left(T_{a}\right)=\mathcal{M}_{a}{ }^{b} T_{b}$.

Since $\operatorname{det}(M)=b^{10} e^{6}$ is not allowed to be zero, we conclude that $b \neq 0$ and $e \neq 0$, which decomposes the eight-parameter family into four connected components. Notice that the matrices $\mathcal{M}$ such that $a=c=d=f=g=h=0$ and $b, e \in\{ \pm 1\}$ form a subgroup of Aut $(\mathfrak{l})$ isomorphic to $\mathbb{Z}_{2} \times \mathbb{Z}_{2}$. Its action on $\mathfrak{l}$ is diagonal as indicated in the theorem, and can be read off from (4.12).

\subsection{Identifying the open orbit corresponding to Time-Space reduction}

Under automorphisms, the Gram matrix $\mathcal{G}_{1}$ given by (4.9) transforms according to

$$
\mathcal{G}_{1} \mapsto \mathcal{M} \mathcal{G}_{1} \mathcal{M}^{T}
$$

where $\mathcal{M}$ is the matrix (4.12) representing the dual of a general automorphism of the Iwasawa algebra $\mathfrak{l}^{\prime}$. We now impose that the transformed Gram matrix is diagonal up to scale. The related calculations can be easily performed using Maple. By imposing successively the vanishing of off-diagonal entries of the transformed Gram matrix, one obtains constraints on the eight parameters of the automorphism. The parameters have to take the values

$$
d=-\frac{1}{\sqrt{3}}, \quad f=-\frac{1}{2}, \quad h=g=0, \quad a=-\frac{1}{6}, \quad c=-\frac{1}{2}, \quad b= \pm \frac{1}{2}, \quad e= \pm \frac{2}{\sqrt{3}} .
$$

This shows that there is a unique inner automorphism $(b, e>0)$ which diagonalizes the Gram matrix, and as well a unique such automorphism in each component of Aut $(\mathfrak{l})$. The diagonalized Gram matrix is in all cases

$$
\mathcal{G}_{1}^{\text {diag }}=\frac{1}{2} \operatorname{diag}(-1,1,-1,1,-1,1,1-1) .
$$

This agrees, up to an overall (positive) factor, with the metric (2.9), (2.13) of the scalar manifold $M^{(T S)}$ obtained by TS reduction $\left(\epsilon_{1}=1, \epsilon_{2}=-1\right)$. We have therefore shown: 
Proposition 7 The left-invariant metric $g^{(T S)}=g^{(1,-1)}$ on L obtained by dimensional reduction of five-dimensional minimal pure supergravity is related by a unique inner automorphism combined with a re-scaling by a factor of $\frac{1}{2}$ to the left-invariant metric $g_{1}$ on $L$ obtained from the open orbit $M_{1}=L^{\prime} \cdot o \subset S=G / G_{e v}$, where $L^{\prime}=C_{a}(L)$, $a=\exp \left(e^{1}+e_{3}^{1}\right) \in G$, is the Iwasawa subgroup constructed in subsection 4.1.

\subsection{Identifying the open orbit corresponding to Space-Time reduction}

Next, we look for another $a \in G$ such that the Iwasawa subalgebra $\mathfrak{l}^{\prime \prime}=\operatorname{Ad}_{a}(\mathfrak{l}) \subset \mathfrak{g}$, is transversal to $\mathfrak{g}_{e v}$, and hence gives rise to a second open orbit $M_{2}=L^{\prime \prime} \cdot o \subset S$, where $L^{\prime \prime}=\exp \left(\mathfrak{I}^{\prime \prime}\right)$. The aim is to match $M_{2}$ with $M^{(S T)}$, up to a covering, using again an inner automorphism of $L$ to relate the corresponding left-invariant metrics $g_{2}=\varphi_{2}^{*} g_{S}$ and $g^{(S T)}$ on $L$. Here $\varphi_{2}: L \rightarrow M_{2}$ is the covering $x \mapsto C_{a}(x) \cdot o$. This procedure involves choosing $\xi \in \mathfrak{g}$ such that $a=\exp (\xi)$ has the desired properties. Investigating candidates for $\xi$ is tedious but manageable using Maple. Otherwise we follow the same steps as for $\mathfrak{l}^{\prime}$.

We use the following basis of $\mathfrak{g}$ :

$$
\begin{aligned}
& b_{1}=e_{1}^{1}-e_{2}^{2}, \quad b_{2}=e_{2}^{2}-e_{3}^{3}, \quad b_{3}=e_{1}^{2}, \quad b_{4}=e_{1}^{3}, \quad b_{5}=e_{2}^{3}, \quad b_{6}=e_{2}^{1}, \quad b_{7}=e_{3}^{1}, \\
& b_{8}=e_{3}^{2}, \quad b_{9}=e_{1}, \quad b_{10}=e_{2}, \quad b_{11}=e_{3}, \quad b_{12}=e^{1}, \quad b_{13}=e^{2}, \quad b_{14}=e^{3} .
\end{aligned}
$$

Note that $\mathfrak{l}=\operatorname{span}\left\{b_{1}, b_{2}, b_{3}, b_{4}, b_{5}, b_{9}, b_{13}, b_{14}\right\}$, with the relation to the basis $\left(\mathcal{V}_{b}\right)$ given by (3.2). We take $\xi=e_{3}^{2}+e^{1}$ and compute $X^{\prime}=\operatorname{Ad}_{a} X$, where $a=\exp \xi$, for all basis elements $X=b_{m}$ of $\mathfrak{l}$ :

$$
\begin{aligned}
b_{1}^{\prime} & =b_{1}-b_{8}+b_{12}, \quad b_{2}^{\prime}=b_{2}+2 b_{8}, \quad b_{3}^{\prime}=b_{3}-b_{7}+b_{11}+b_{13}, \\
b_{4}^{\prime} & =-b_{3}+b_{4}+b_{6}+b_{7}-b_{10}-b_{11}-b_{13}+b_{14}, \\
b_{5}^{\prime} & =-b_{2}+b_{5}-b_{8}, \quad b_{9}^{\prime}=-2 b_{1}-b_{2}+b_{9}-b_{12}, \\
b_{13}^{\prime} & =-3 b_{7}+2 b_{11}+b_{13}, \quad b_{14}^{\prime}=3 b_{6}+3 b_{7}-2 b_{10}-2 b_{11}-b_{13}+b_{14} .
\end{aligned}
$$

As before we denote by $\varphi$ the composition $\pi \circ \operatorname{Ad}_{a}$ where $\pi: \mathfrak{g} \rightarrow \mathfrak{g}_{\text {odd }}$ is the projection along $\mathfrak{g}_{e v}$. Using the above formulae we apply $\varphi$ to the basis elements $\mathcal{V}_{b}$ and express the result in the basis $\left(f_{1}, \ldots, f_{8}\right):=\left(b_{9}, b_{10}, b_{12}, b_{13}, b_{4}, b_{5}, b_{7}, b_{8}\right)$ of $\mathfrak{g}_{\text {odd }}$. For example

$$
\varphi\left(\mathcal{V}_{1}\right)=-3 \varphi\left(b_{4}\right)=-3 b_{4}-3 b_{7}+3 b_{10}+3 b_{13} .
$$

The result is summarized by the matrix $A$, which is the transpose of the matrix representing $\varphi: \mathfrak{l} \rightarrow \mathfrak{g}_{\text {odd }}$ with respect to the bases $\left(\mathcal{V}_{b}\right)$ and $\left(f_{b}\right)$, that is $\varphi\left(\mathcal{V}_{b}\right)=A_{b c} f_{c}$ :

$$
A=\left(\begin{array}{cccccccc}
0 & 3 & 0 & 3 & -3 & 0 & -3 & 0 \\
0 & 0 & \frac{1}{2} & 0 & 0 & 0 & 0 & \frac{1}{2} \\
0 & 0 & 0 & \frac{1}{\sqrt{3}} & 0 & 0 & -\sqrt{3} & 0 \\
0 & 0 & \frac{1}{2 \sqrt{3}} & 0 & 0 & 0 & 0 & -\frac{\sqrt{3}}{2} \\
0 & 0 & 0 & 0 & 0 & \sqrt{3} & 0 & -\sqrt{3} \\
0 & -2 & 0 & -1 & 0 & 0 & 3 & 0 \\
0 & 0 & 0 & -\sqrt{3} & 0 & 0 & \sqrt{3} & 0 \\
-1 & 0 & 1 & 0 & 0 & 0 & 0 & 0
\end{array}\right) .
$$


One checks that $\operatorname{det}(A)=-12 \neq 0$, and therefore the vectors $\varphi\left(\mathcal{V}_{b}\right)$ are linearly independent, and $\mathfrak{l}^{\prime \prime}=\operatorname{span}\left\{\mathcal{V}_{b}\right\} \simeq \mathfrak{g}_{\text {odd }}$ is transversal. The Gram matrix $\mathcal{G}_{2}$ of the scalar product $\langle\cdot, \cdot\rangle_{2}=\varphi^{*}\langle\cdot, \cdot\rangle_{B}$ on $\mathfrak{l}$ with respect to the basis $\left(\mathcal{V}_{b}\right)$ is given by

$$
\mathcal{G}_{2}=A \mathcal{G} A^{T}
$$

where $\mathcal{G}$ is the Gram matrix of the scalar product $\langle\cdot, \cdot\rangle_{B}$ on $\mathfrak{g}_{\text {odd }}$ with respect to the basis $\left(f_{b}\right)$, as computed in Lemma 1 . The resulting matrix is

$$
\mathcal{G}_{2}=\left(\begin{array}{cccccccc}
72 & 0 & 6 \sqrt{3} & 0 & 0 & -36 & -12 \sqrt{3} & 0 \\
0 & 0 & 0 & 0 & \frac{\sqrt{3}}{2} & 0 & 0 & -\frac{3}{2} \\
6 \sqrt{3} & 0 & 0 & 0 & 0 & -2 \sqrt{3} & 0 & 0 \\
0 & 0 & 0 & 0 & -\frac{3}{2} & 0 & 0 & -\frac{\sqrt{3}}{2} \\
0 & \frac{\sqrt{3}}{2} & 0 & -\frac{3}{2} & -6 & 0 & 0 & 0 \\
-36 & 0 & -2 \sqrt{3} & 0 & 0 & 12 & 6 \sqrt{3} & 0 \\
-12 \sqrt{3} & 0 & 0 & 0 & 0 & 6 \sqrt{3} & 0 & 0 \\
0 & -\frac{3}{2} & 0 & -\frac{\sqrt{3}}{2} & 0 & 0 & 0 & -6
\end{array}\right) .
$$

Now we apply a general automorphism of $\mathfrak{l}$ with matrix $\mathcal{M}$ as in (4.12) and impose that $\mathcal{N G}_{2} \mathcal{M}^{T}$ is diagonal up to scale. This leads to the following constraints on the parameters of $\mathcal{M}$ :

$$
f=0, \quad d=\frac{1}{12} \sqrt{3}, \quad h=-\frac{1}{4}, \quad g=0, \quad a=0, \quad c=0, \quad b= \pm 1, \quad e= \pm \frac{1}{2 \sqrt{3}} .
$$

Thus there is again a unique inner automorphism diagonalizing the Gram matrix, and precisely one such automorphism in each component of Aut(l). The diagonalized Gram matrix is in all cases

$$
\mathcal{G}_{2}^{\text {diag }}=\frac{1}{2} \operatorname{diag}(1,1,1,1,-1,-1,-1,-1)
$$

which agrees, up to an overall (positive) scale factor with the metric $g^{(S T)}(2.9),(2.13)$ of the scalar manifold $M^{(S T)}$ obtained by ST reduction, $\left(\epsilon_{1}=-1, \epsilon_{2}=1\right)$. Thus we have shown:

Proposition 8 The left-invariant metric $g^{(S T)}=g^{(-1,1)}$ on L obtained by dimensional reduction of five-dimensional minimal pure supergravity is related by a unique inner automorphism combined with a re-scaling by a factor of $\frac{1}{2}$ to the left-invariant metric $g_{2}$ on $L$ obtained from the open orbit $M_{2}=L^{\prime \prime} \cdot o \subset S=G / G_{e v}$, where $L^{\prime \prime}=C_{a}(L)$, $a=\exp \left(e_{3}^{2}+e^{1}\right) \in G$, is the Iwasawa subgroup constructed above.

We also have:

Proposition 9 The left-invariant metrics $g^{(T S)}$ and $g^{(S T)}$ (equivalently $g_{1}$ and $g_{2}$ ) on $L$ are not related by any automorphism of $L$. 
Proof: This can be proven by showing that an automorphism cannot transform the diagonal Gram matrix $\mathcal{G}_{1}^{\text {diag }}(4.13)$ of $g^{(T S)}$ to the diagonal Gram matrix $\mathcal{G}_{2}^{\text {diag }}(4.15)$ of $g^{(S T)}$. If such an automorphism existed, then there would exist a matrix $\mathcal{M}$ of the form (4.12) such that $\mathcal{M Y}_{1}^{\text {diag }} \mathcal{M}^{T}=\mathcal{G}_{2}^{\text {diag }}$. Analysing the conditions imposed on the parameters, one finds $a=d=f=g=h=0$, which in turn implies (amongst other things) that $\Omega\left(\eta^{2}\right)=b^{3} e^{2} \eta^{2}$. Hence we see that we need $b^{6} e^{4}=-1$ which is impossible.

Alternatively, this follows from the uniqueness of the diagonalization of the Gram matrices $\mathcal{G}_{1}$ and $\mathcal{G}_{2}$ by automorphisms, which was observed above.

In the next section we will investigate the geometry of the manifolds $\left(L, g_{1}\right)$ and $\left(L, g_{2}\right)$ more closely.

\section{Geometric structures on the Iwasawa subgroup of $G_{2(2)}$}

We now explore the geometrical structures carried by the Lie algebra $\mathfrak{l}$ of the Iwasawa subgroup $L$, equipped with the three metrics related to dimensional reductions of minimal pure five-dimensional supergravity, and find explicit expressions for the (para-)quaternionic structures, the Levi-Civita connection and the curvature.

Recall that the Lie algebra $\mathfrak{l}$ is equipped with the basis $\left(\mathcal{V}_{a}\right)$ defined in (3.2) and the left-invariant metrics $g_{1}, g_{2}$ with the Gram matrices $\mathcal{G}_{1}, \mathcal{G}_{2}$ given in (4.9) and (4.14). As observed above there exists in both cases a unique inner automorphism of $\mathfrak{l}$ transforming the above Gram matrices into diagonal forms given by (4.13), (4.15). Let us denote in both cases by $\left(T_{a}\right)=\left(V_{2}, U^{2}, A, B, V_{0}, V_{1}, U^{0}, U^{1}\right)$ the, up to scale, orthonormal basis which corresponds to the basis $\left(\mathcal{V}_{a}\right)$ under this unique inner automorphism. For convenience, in this section we work with rescaled metrics on $L$ and corresponding scalar products on $\mathfrak{l}$, for which $\left(T_{a}\right)$ is an orthonormal basis. For completeness we also consider the case of SS reduction which corresponds to a positive definite metric on $L$. Thus the Gram matrices with respect to the basis $\left(T_{a}\right)$ for the three cases are:

$$
\mathcal{G}^{\text {diag }}=\left(-\epsilon_{1}, 1,-\epsilon_{1}, 1,-\epsilon,-\epsilon_{2},-\epsilon_{2},-\epsilon\right)
$$

The corresponding metric on $L$ is denoted $\bar{g}=\bar{g}^{\left(\epsilon_{1}, \epsilon_{2}\right)}$ and the associated scalar product on $\mathfrak{l}$ by $\langle\cdot, \cdot\rangle=\langle\cdot, \cdot\rangle^{\epsilon_{1}, \epsilon_{2}}$.

Summarizing we are given the Lie algebra $\mathfrak{l}$ with the basis

$$
\left(T_{a}\right)=\left(V_{2}, U^{2}, A, B, V_{0}, V_{1}, U^{0}, U^{1}\right)
$$

with structure constants (2.12) and a pseudo-Euclidean scalar product $\langle\cdot, \cdot\rangle$ defined by the Gram matrix (5.1) with respect to the basis $\left(T_{a}\right)$. We now state the main results which will be proved in this section. 
We first define the following skew-symmetric endomorphisms.

$$
\begin{aligned}
J_{1}= & \epsilon_{2} U^{2} \wedge V_{2}-B \wedge A+\epsilon \frac{\sqrt{3}}{2} U^{1} \wedge U^{0}-\epsilon_{2} \frac{1}{2} U^{1} \wedge V_{1} \\
& +\epsilon_{2} \frac{1}{2} U^{0} \wedge V_{0}+\epsilon \frac{\sqrt{3}}{2} V_{1} \wedge V_{0}, \\
J_{2}= & \epsilon_{2} \frac{\sqrt{3}}{2} U^{1} \wedge V_{2}+\epsilon \frac{1}{2} V_{0} \wedge V_{2}-\frac{1}{2} U^{0} \wedge U^{2}-\epsilon_{1} \frac{\sqrt{3}}{2} V_{1} \wedge U^{2} \\
& -\frac{1}{2} U^{1} \wedge A-\epsilon_{1} \frac{\sqrt{3}}{2} V_{0} \wedge A-\frac{\sqrt{3}}{2} U^{0} \wedge B+\epsilon_{1} \frac{1}{2} V_{1} \wedge B, \\
J_{3}= & \epsilon_{2} \frac{1}{2} U^{0} \wedge V_{2}-\epsilon \frac{\sqrt{3}}{2} V_{1} \wedge V_{2}-\epsilon_{1} \frac{\sqrt{3}}{2} U^{1} \wedge U^{2}+\frac{1}{2} V_{0} \wedge U^{2} \\
& -\frac{\sqrt{3}}{2} U^{0} \wedge A+\epsilon_{1} \frac{1}{2} V_{1} \wedge A-\epsilon_{1} \frac{1}{2} U^{1} \wedge B-\frac{\sqrt{3}}{2} V_{0} \wedge B, \\
\tilde{J}_{1}= & -\epsilon_{2} U^{2} \wedge V_{2}-B \wedge A+\epsilon \frac{\sqrt{3}}{2} U^{1} \wedge U^{0}-\epsilon_{2} \frac{1}{2} U^{1} \wedge V_{1} \\
& +\epsilon_{2} \frac{1}{2} U^{0} \wedge V_{0}+\epsilon \frac{\sqrt{3}}{2} V_{1} \wedge V_{0} .
\end{aligned}
$$

Here we use the following standard identification of bi-vectors with skew-symmetric endomorphisms:

$$
(u \wedge v)(w)=u\langle v, w\rangle-\langle u, w\rangle v, \quad u, v, w, \in \mathfrak{l}
$$

Proposition 10 The endomorphisms $J_{\alpha}$ of $\mathfrak{l}$ are pairwise anti-commuting and satisfy the following relations: ${ }^{3}$

$$
\left(J_{1}\right)^{2}=\epsilon_{1} I d, \quad\left(J_{2}\right)^{2}=\epsilon_{2} I d, \quad\left(J_{3}\right)^{2}=\epsilon_{3} I d:=\epsilon I d, \quad J_{3}=J_{1} J_{2} .
$$

Proof: This follows by direct calculation.

Notice that the endomorphisms $J_{\alpha}$ define left-invariant skew-symmetric almost $\epsilon_{\alpha^{-}}$ complex $^{4}$ structures on the Lie group $L$, which will be denoted by the same symbols. We put $Q:=\operatorname{span}\left\{J_{\alpha} \mid \alpha=1,2,3\right\}$.

Theorem $2(L, \bar{g}, Q)$ is an $\epsilon$-quaternionic-Kähler manifold with left-invariant $\epsilon$ quaternionic structure $Q$, and $J_{1}, \tilde{J}_{1}$ are integrable left-invariant skew-symmetric $\epsilon_{1}$ complex structures on $(L, \bar{g})$.

Proof: The integrability of the structures $J_{1}, \tilde{J}_{1}$ is proven by computation of the Nijenhuis tensor $^{5}$

$$
N_{J}(X, Y)=-J^{2}[X, Y]+J[J X, Y]+J[X, J Y]-[J X, J Y]=0,
$$

\footnotetext{
${ }^{3}$ Recall that $\epsilon=-\epsilon_{1} \epsilon_{2}$.

${ }^{4}$ By the terminology " $\epsilon$-complex", " $\epsilon$-quaternionic", etc. we mean "complex", "quaternionic", etc. if $\epsilon=-1$ and "para-complex", "para-quaternionic", etc. if $\epsilon=1$.

${ }^{5}$ Notice that by the Newlander-Nirenberg theorem and the Frobenius theorem, respectively, the vanishing of the Nijenhuis tensor $N_{J}$ of an almost $\epsilon$-complex structure $J$ on a smooth manifold $M$ implies that $J$ defines on $M$ the structure of a complex, respectively para-complex, manifold.
} 
for $J=J_{1}$ and $J=\tilde{J}_{1}$ using the formulae (2.12), (5.3) and (5.6). One also finds that $N_{J_{2}}, N_{J_{3}}$ do not vanish. To prove the $\epsilon$-quaternionic-Kähler property we need to check that $Q$ is parallel. The explicit expression for the Levi-Civita connection is given in formula (5.9) in subsection 5.1. Using this formula, it is checked in Proposition 12. that $Q$ is parallel.

The curvature tensor of the $\epsilon$-quaternionic-Kähler manifold $(L, \bar{g}, Q)$ is given in formula (5.12) in subsection 5.2. Based on the formulae for the Levi-Civita connection and its curvature we have verified by explicit calculation that the curvature tensor is parallel. This provides a second, independent proof of the fact, established in section 4, that the manifold $(L, \bar{g})$ is locally symmetric.

For $\epsilon_{1}=\epsilon_{2}=-1$ Theorem 2 recovers Alekseevsky's description [10] of the symmetric quaternionic-Kähler manifold of non-compact type $G_{2(2)} / S_{4}$ as a solvable Lie group $L$ endowed with a left-invariant quaternionic-Kähler structure. For completeness we include in subsection 5.3 a discussion relating our approach with Alekseevsky's description in terms of representations of Kählerian Lie algebras.

\subsection{Computation of the Levi Civita connection}

To compute the Levi-Civita connection of a pseudo-Riemannian metric $g$, we use the Koszul formula

$$
\begin{aligned}
2 g\left(\nabla_{X} Y, Z\right)= & X g(Y, Z)+Y g(X, Z)-Z g(X, Y) \\
& +g([X, Y], Z)-g(X,[Y, Z])-g(Y,[X, Z]),
\end{aligned}
$$

where $X, Y, Z$ are vector fields. ${ }^{6}$ For a left-invariant metric on a Lie group $L$ the vector fields $X, Y, Z$ can be taken to be left-invariant and therefore correspond to vectors in the Lie algebra $\mathfrak{l}$, in which case the first three terms on the right hand side vanish. The computation of the Levi-Civita connection is thus reduced to computing commutators and scalar products of vectors in $\mathfrak{l}$. Notice that the covariant derivative $\nabla_{X}$ acts on $\mathfrak{l}$ as an endomorphism, which satisfies

$$
T(X, Y)=\nabla_{X} Y-\nabla_{Y} X-[X, Y]=0, \quad \forall X, Y \in \mathfrak{l},
$$

and which is skew as a consequence of the metric compatibility of the Levi-Civita connection. Therefore we can express $\nabla_{X}$ as a wedge product of generators, using the convention (5.7).

Using the commutators (2.12) in the solvable Lie algebra $\mathfrak{l}$ together with the fact that the generators $T_{a}$ form an orthonormal basis (5.2) with the Gram matrix (5.1) it is straightforward to obtain the following result.

\footnotetext{
${ }^{6}$ When taking $X, Y, Z$ to be coordinate vector fields (and hence to commute), the last three terms on the right hand side vanish and one recovers the usual formula for the Levi-Civita connection in terms of Christoffel symbols.
} 
Proposition 11 The Levi-Civita connection $\nabla$ of $(L, \bar{g})$ is given by:

$$
\begin{aligned}
\nabla_{V_{2}}= & U^{2} \wedge V_{2}+\frac{1}{2} U^{0} \wedge V_{0}+\frac{1}{2} U^{1} \wedge V_{1}, \\
\nabla_{U^{2}}= & 0, \\
\nabla_{A}= & \frac{1}{\sqrt{3}} B \wedge A+\frac{1}{2} \epsilon V_{0} \wedge V_{1}+\frac{1}{2} \epsilon U^{0} \wedge U^{1}+\frac{1}{\sqrt{3}} \epsilon_{2} U^{1} \wedge V_{1}, \\
\nabla_{B}= & 0, \\
\nabla_{V_{0}}= & -\frac{1}{2} V_{0} \wedge U^{2}-\frac{1}{2} \epsilon_{2} U^{0} \wedge V_{2}+\frac{\sqrt{3}}{2} V_{0} \wedge B+\frac{1}{2} \epsilon_{1} V_{1} \wedge A, \\
\nabla_{V_{1}}= & -\frac{1}{2} V_{1} \wedge U^{2}-\frac{1}{2} \epsilon U^{1} \wedge V_{2}+\frac{1}{2 \sqrt{3}} V_{1} \wedge B \\
& -\frac{1}{2} V_{0} \wedge A-\frac{1}{\sqrt{3}} \epsilon_{1} U^{1} \wedge A, \\
\nabla_{U^{0}}= & -\frac{1}{2} U^{0} \wedge U^{2}+\frac{1}{2} \epsilon V_{0} \wedge V_{2}-\frac{\sqrt{3}}{2} U^{0} \wedge B+\frac{1}{2} U^{1} \wedge A, \\
\nabla_{U^{1}}= & -\frac{1}{2} U^{1} \wedge U^{2}+\frac{1}{2} \epsilon_{2} V_{1} \wedge V_{2}-\frac{1}{2 \sqrt{3}} U^{1} \wedge B \\
& -\frac{1}{2} \epsilon_{1} U^{0} \wedge A+\frac{1}{\sqrt{3}} V_{1} \wedge A .
\end{aligned}
$$

For illustration, the first line of the above formula is equivalent to:

$$
\begin{aligned}
& \nabla_{V_{2}} V_{2}=-\epsilon_{1} U^{2}, \quad \nabla_{V_{2}} U_{2}=-V_{2}, \quad \nabla_{V_{2}} A=0, \quad \nabla_{V_{2}} B=0 . \\
& \nabla_{V_{2}} V_{0}=-\frac{1}{2} \epsilon U^{0}, \quad \nabla_{V_{2}} U^{0}=\frac{1}{2} \epsilon_{2} V_{0}, \quad \nabla_{V_{2}} V_{1}=-\frac{1}{2} \epsilon_{2} U^{1}, \quad \nabla_{V_{2}} U^{1}=\frac{1}{2} \epsilon V_{1} .
\end{aligned}
$$

The covariant derivatives of the structures $J_{\alpha}$ can now be computed by taking commutators between the corresponding skew endomorphisms $\nabla_{X}$ and $J_{\alpha}$ for all $X \in \mathfrak{l}$. We note the following useful formula:

$$
[X \wedge Y, Z \wedge W]=(X \wedge W)\langle Y, Z\rangle+(Y \wedge Z)\langle X, W\rangle-(X \wedge Z)\langle Y, W\rangle-(Y \wedge W)\langle X, Z\rangle .
$$

Using Proposition 11 and the explicit expressions for the structures $J_{\alpha}$ given in (5.3)-(5.5), we obtain:

\section{Proposition 12}

$$
\begin{aligned}
& {\left[\nabla_{X}, J_{1}\right]=\hat{\alpha}(X) J_{2}+\hat{\beta}(X) J_{3},} \\
& {\left[\nabla_{X}, J_{2}\right]=\epsilon \hat{\alpha}(X) J_{1}+\hat{\gamma}(X) J_{3},} \\
& {\left[\nabla_{X}, J_{3}\right]=\epsilon_{2} \hat{\beta}(X) J_{1}+\epsilon_{1} \hat{\gamma}(X) J_{2},}
\end{aligned}
$$

where $\hat{\alpha}, \hat{\beta}, \hat{\gamma} \in \mathfrak{l}^{*}$ are one-forms, which are related to the dual basis (2.7) of the basis $\left(T_{a}\right)$ of $\mathfrak{l}$ by

$$
\hat{\alpha}=-\frac{1}{2} \eta^{0}-\frac{\sqrt{3}}{2} \xi_{1}, \quad \hat{\beta}=-\epsilon_{1} \frac{\sqrt{3}}{2} \eta^{1}-\frac{1}{2} \xi_{0}, \quad \hat{\gamma}=\epsilon_{2} \frac{1}{2} \eta^{2}-\frac{\sqrt{3}}{2} \alpha .
$$

This shows that the $\epsilon$-quaternionic structure $Q$ is parallel. 


\subsection{Curvature}

Given the Levi-Civita connection it is straightforward to compute the curvature by the defining formula

$$
R(X, Y)=\left[\nabla_{X}, \nabla_{Y}\right]-\nabla_{[X, Y]},
$$

where $X, Y$ are vector fields. In our setting we take $X, Y$ to be left-invariant vector fields on the Lie group $L$ and identify them with elements of $\mathfrak{l}$. Then $R(X, Y)$ is considered as a skew-symmetric endomorphism of $\mathfrak{l}$.

Proposition 13 The curvature endomorphisms of $(L, \bar{g})$ are given by:

$$
\begin{aligned}
R\left(U^{2}, V_{2}\right) & =-\nabla_{V_{2}}, \quad R\left(U^{2}, B\right)=R\left(U^{2}, A\right)=0 \\
R\left(U^{2}, V_{0}\right) & =-\frac{1}{2} \nabla_{V_{0}}, \quad R\left(U^{2}, V_{1}\right)=-\frac{1}{2} \nabla_{V_{1}}, \quad R\left(U^{2}, U^{0}\right)=-\frac{1}{2} \nabla_{U^{0}}, \\
R\left(U^{2}, U^{1}\right) & =-\frac{1}{2} \nabla_{U^{1}}, \\
R\left(V_{2}, B\right) & =R\left(V_{2}, A\right)=0, \quad R\left(V_{2}, V_{0}\right)=\frac{1}{2} \epsilon \nabla_{U^{0}}, \\
R\left(V_{2}, V_{1}\right) & =\frac{1}{2} \epsilon_{2} \nabla_{U^{1}}, \quad R\left(V_{2}, U^{0}\right)=-\frac{1}{2} \epsilon_{2} \nabla_{V_{0}}, \quad R\left(V_{2}, U^{1}\right)=-\frac{1}{2} \epsilon \nabla_{V_{1}}, \\
R(B, A) & =-\frac{1}{\sqrt{3}} \nabla_{A}, \quad R\left(B, V_{0}\right)=\frac{\sqrt{3}}{2} \nabla_{V_{0}}, \quad R\left(B, V_{1}\right)=\frac{1}{2 \sqrt{3}} \nabla_{V_{1}}, \\
R\left(B, U^{0}\right) & =-\frac{\sqrt{3}}{2} \nabla_{U^{0}}, \quad R\left(B, U^{1}\right)=-\frac{1}{2 \sqrt{3}} \nabla_{U^{1}}, \\
R\left(A, V_{0}\right) & =-\frac{1}{2} \nabla_{V_{1}}, \quad R\left(A, V_{1}\right)=\frac{1}{2} \epsilon_{1} \nabla_{V_{0}}+\frac{1}{\sqrt{3}} \nabla_{U^{1}}, \\
R\left(A, U^{0}\right) & =-\frac{1}{2} \epsilon_{1} \nabla_{U^{1}}, \quad R\left(A, U^{1}\right)=\frac{1}{2} \nabla_{U^{0}}-\frac{1}{\sqrt{3}} \epsilon_{1} \nabla_{V_{1}}, \\
R\left(V_{0}, V_{1}\right) & =-\frac{1}{2} \epsilon \nabla_{A}, \quad R\left(V_{0}, U^{0}\right)=\frac{1}{2} \nabla_{V_{2}}, \quad R\left(V_{0}, U^{1}\right)=0 \\
R\left(V_{1}, U^{0}\right) & =0, \quad R\left(V_{1}, U^{1}\right)=\frac{1}{2} \nabla_{V_{2}}+\frac{1}{\sqrt{3}} \epsilon_{2} \nabla_{A}, \quad R\left(U^{0}, U^{1}\right)=-\frac{1}{2} \epsilon \nabla_{A} .
\end{aligned}
$$

From a tedious but straightforward calculation we deduce:

Corollary 1 The pseudo-Riemannian manifold $(L, \bar{g})$ is locally symmetric:

$$
\nabla R=0
$$

\subsection{Relation with $Q$-representations of Kählerian Lie algebras in the Rieman- nian case}

In this section we explain the relation between our construction and Alekseevsky's classification of left-invariant quaternionic-Kähler structures on solvable Lie groups using the so-called $Q$-representations of Kählerian Lie algebras [10]. In the Riemannian case of our 
construction, $\epsilon_{1}=\epsilon_{2}=-1$, we can make the following unitary basis transformation of the Lie algebra:

$$
\begin{aligned}
G_{0}:=V_{2}, \quad H_{0}:=U^{2}, \quad G_{1}:=A, \quad H_{1}:=B, \\
\tilde{G}_{0}:=-\frac{\sqrt{3}}{2} U^{1}-\frac{1}{2} V_{0}, \quad \tilde{G}_{1}:=-\frac{1}{2} U^{1}+\frac{\sqrt{3}}{2} V_{0}, \\
\tilde{H}_{0}:=-\frac{1}{2} U^{0}+\frac{\sqrt{3}}{2} V_{1}, \quad \tilde{H}_{1}:=-\frac{\sqrt{3}}{2} U^{0}-\frac{1}{2} V_{1} .
\end{aligned}
$$

In this new orthonormal basis, the complex structures take the following simple form:

$$
\begin{aligned}
& J_{1}=-H_{0} \wedge G_{0}-H_{1} \wedge G_{1}+\tilde{H}_{0} \wedge \tilde{G}_{0}+\tilde{H}_{1} \wedge \tilde{G}_{1}, \\
& J_{2}=\tilde{G}_{0} \wedge G_{0}+\tilde{H}_{0} \wedge H_{0}+\tilde{G}_{1} \wedge G_{1}+\tilde{H}_{1} \wedge H_{1}, \\
& J_{3}=-\tilde{G}_{0} \wedge H_{0}+\tilde{H}_{0} \wedge G_{0}-\tilde{G}_{1} \wedge H_{1}+\tilde{H}_{1} \wedge G_{1}, \\
& \tilde{J}_{1}=H_{0} \wedge G_{0}-H_{1} \wedge G_{1}+\tilde{H}_{0} \wedge \tilde{G}_{0}+\tilde{H}_{1} \wedge \tilde{G}_{1} .
\end{aligned}
$$

In Alekseevsky's construction, when applied to the special case of $(L, \bar{g}) \cong G_{2(2)} / S O_{4}$, one starts with the sum of two elementary Kählerian Lie algebras

$$
\mathfrak{u}=\mathfrak{f}_{0} \oplus \mathfrak{f}_{1},
$$

where

$$
\begin{array}{ll}
\mathfrak{f}_{0}=\operatorname{span}\left\{G_{0}, H_{0}\right\}, & {\left[H_{0}, G_{0}\right]=G_{0},} \\
\mathfrak{f}_{1}=\operatorname{span}\left\{G_{1}, H_{1}\right\}, & {\left[H_{1}, G_{1}\right]=\frac{1}{\sqrt{3}} G_{1} .}
\end{array}
$$

The corresponding Lie group $U$ acts simply transitively on the product of two complex hyperbolic lines with curvatures -1 and $-\frac{1}{3}$, respectively. The latter is the projective special Kähler manifold obtained by applying the local $r$-map to a zero-dimensional manifold. The symmetric space corresponding to the complex hyperbolic line is $\mathrm{SU}(1,1) / \mathrm{U}(1) \simeq$ $\mathrm{SL}(2, \mathbb{R}) / \mathrm{SO}(2)$.

One then chooses a certain representation $T: \mathfrak{u} \rightarrow \mathfrak{g l}(\tilde{\mathfrak{u}})$ and extends the Lie algebra $\mathfrak{u}$ to a solvable Lie algebra $\mathfrak{l}=\mathfrak{u} \oplus \tilde{\mathfrak{u}}$, with $[\tilde{\mathfrak{u}}, \tilde{\mathfrak{u}}] \subset \mathfrak{u}$. The representation space $\tilde{\mathfrak{u}}$ is related to $\mathfrak{u}$ by an isomorphism of vector spaces $\mathfrak{u} \rightarrow \tilde{\mathfrak{u}}, X \mapsto \tilde{X}$. The above basis is consistent with this isomorphism, i.e. $G_{0}$ is mapped to $\tilde{G}_{0}$, etc. The first complex structure $J_{1}$ on $\mathfrak{l}$ is then determined by the condition that the restriction $\left.J_{1}\right|_{\mathfrak{u}}$ is the natural complex structure on the Kählerian Lie algebra $\mathfrak{u}$, together with the property that $J_{1} \tilde{X}=-\widetilde{J_{1} X}$ for all $X \in \mathfrak{u}$. The second complex structure is defined by $J_{2} X=\tilde{X}$ and $J_{2} \tilde{X}=-X$ for all $X \in \mathfrak{u}$. The representation $T$ is chosen as a $Q$-representation, which means that it satisfies certain conditions which ensure that $\left(J_{1}, J_{2}, J_{3}=J_{1} J_{2}\right)$ is a quaternionic-Kähler structure on the solvable Lie algebra $\mathfrak{l}$.

One possible alternative approach to our work on para-quaternionic-Kähler structures would have been to adapt Alekseevsky's method using bases analogous to the basis $\left(G_{0}, G_{1}, H_{0}, H_{1}, \tilde{G}_{0}, \tilde{G}_{1}, \tilde{H}_{0}, \tilde{H}_{1}\right)$. However, the basis would have needed to be adapted to 
the different scalar products, so that we would have needed to work with three different bases, depending on the values of $\epsilon_{1}$ and $\epsilon_{2}$. The advantage of the basis $\mathcal{T}$ is that it can be used in all three cases. Moreover, this basis is natural from the point of view of dimensional reduction in supergravity.

\subsection{Conjugate Iwasawa subgroups vs disjoint open $L$-orbits}

For completeness we will explain the relation between, on the one hand, the open orbits $M^{\prime}=L^{\prime} \cdot o$ of the canonical base point $o \in S=G / H$ under subgroups $L^{\prime}=C_{a}(L)=$ $a L a^{-1} \subset G(a \in G)$ conjugate to the standard Iwasawa subgroup $L \subset G$ and, on the other hand, the $L$-orbits $L \cdot o^{\prime}$ of different points $o^{\prime} \in S$. Notice that the orbits $M^{\prime}=L^{\prime} \cdot o$ and $L \cdot o^{\prime}, o^{\prime}=a^{-1} o$, are related by

$$
M^{\prime}=L_{a}\left(L \cdot o^{\prime}\right),
$$

where $L_{a}: S \rightarrow S$ is the diffeomorphism given by the $G$-action on $S$.

Proposition 14 Let $M^{\prime}=L^{\prime} \cdot o, M^{\prime \prime}=L^{\prime \prime} \cdot o \subset S$ be the two open orbits constructed in sections 4.3 and 4.4. Then the corresponding open $L$-orbits $L \cdot o^{\prime}$ and $L \cdot o^{\prime \prime}$ are disjoint.

Proof: Let us denote by $a^{\prime}, a^{\prime \prime} \in G$ elements such that $L^{\prime}=C_{a^{\prime}}(L), L^{\prime \prime}=C_{a^{\prime \prime}}(L), o^{\prime}=$ $\left(a^{\prime}\right)^{-1} \cdot o$ and $o^{\prime \prime}=\left(a^{\prime \prime}\right)^{-1} \cdot o$. Recall that the open orbits $M^{\prime}$ and $M^{\prime \prime}$ give rise to left-invariant metrics $g^{\prime}=\left(\phi^{\prime}\right)^{*} g_{S}$ and $g^{\prime \prime}=\left(\phi^{\prime \prime}\right)^{*} g_{S}$ on $L$, where $\phi^{\prime}: L \rightarrow M^{\prime}, \phi^{\prime}(x)=C_{a^{\prime}}(x) \cdot o$, and $\phi^{\prime \prime}: L \rightarrow M^{\prime \prime}, \phi^{\prime \prime}(x)=C_{a^{\prime \prime}}(x) \cdot o$, for all $x \in L$. If $L \cdot o^{\prime}$ and $L \cdot o^{\prime \prime}$ are not disjoint, then they coincide, and $o^{\prime \prime} \in L \cdot o^{\prime}$. This means that there exists $a \in L$ such that $o^{\prime \prime}=a o^{\prime}$. Now we show that this implies that the left-invariant metrics $g^{\prime}$ and $g^{\prime \prime}$ on $L$ are related by

$$
g^{\prime \prime}=C_{a^{-1}}^{*} g^{\prime} .
$$

This follows from the equation

$$
\phi^{\prime \prime}=L_{b} \circ \phi^{\prime} \circ C_{a^{-1}}, \quad b=a^{\prime \prime} a\left(a^{\prime}\right)^{-1},
$$

which we will prove below. In fact, using the $G$-invariance of $g_{S},(5.15)$ implies that

$$
g^{\prime \prime}=\left(\phi^{\prime \prime}\right)^{*} g_{S}=\left(C_{a^{-1}}^{*} \circ\left(\phi^{\prime}\right)^{*}\right)\left(L_{b}^{*} g_{S}\right)=\left(C_{a^{-1}}^{*} \circ\left(\phi^{\prime}\right)^{*}\right)\left(g_{S}\right)=C_{a^{-1}}^{*} g^{\prime} .
$$

Now we prove (5.15). We compute for $x \in L$ :

$$
\begin{aligned}
\phi^{\prime}(x) & =C_{a^{\prime}}(x) \cdot o=\left(a^{\prime} x\right) \cdot o^{\prime}, \\
\phi^{\prime \prime}(x) & =C_{a^{\prime \prime}}(x) \cdot o=\left(a^{\prime \prime} x\right) \cdot o^{\prime \prime}=\left(a^{\prime \prime} x a\right) \cdot o^{\prime}=\left(a^{\prime \prime} a C_{a^{-1}}(x)\right) \cdot o^{\prime} \\
& =\left(a^{\prime \prime} a\left(a^{\prime}\right)^{-1} a^{\prime} C_{a^{-1}}(x)\right) \cdot o^{\prime} \stackrel{(5.16)}{=} b \phi^{\prime}\left(C_{a^{-1}}(x)\right) .
\end{aligned}
$$

This proves (5.15) and (5.14), under the assumption that the orbits $L \cdot o^{\prime}$ and $L \cdot o^{\prime \prime}$ are not disjoint. On the other hand, we know from Proposition 9 that $g^{\prime}$ and $g^{\prime \prime}$ are not related by an inner automorphism of $L$. Therefore the orbits are necessarily disjoint.

Corollary 2 The Iwasawa subgroup $L \subset G=G_{2(2)}$ acts with at least two open orbits on $S=G_{2(2)} /(\mathrm{SL}(2) \cdot \mathrm{SL}(2))$. 


\section{Acknowledgements}

This work was partly supported by the German Science Foundation (DFG) under the Collaborative Research Center (SFB) 676 Particles, Strings and the Early Universe.

We would like to thank Owen Vaughan for useful discussions. The work of T.M. is supported in part by STFC grant ST/G00062X/1. The work of P.D. is supported by STFC studentship ST/1505805/1. T.M. thanks the Mathematics Department of the University of Hamburg for Hospitality and Support during various stages of this project. We thank Boris Pioline for bringing reference [4] to our attention.

Open Access. This article is distributed under the terms of the Creative Commons Attribution License (CC-BY 4.0), which permits any use, distribution and reproduction in any medium, provided the original author(s) and source are credited.

\section{References}

[1] G.W. Gibbons and S.W. Hawking, Classification of Gravitational Instanton Symmetries, Commun. Math. Phys. 66 (1979) 291 [INSPIRE].

[2] P. Breitenlohner, D. Maison and G.W. Gibbons, Four-Dimensional Black Holes from Kaluza-Klein Theories, Commun. Math. Phys. 120 (1988) 295 [INSPIRE].

[3] G.W. Moore, Finite in all directions, hep-th/9305139 [INSPIRE].

[4] M. Berkooz and B. Pioline, 5D Black Holes and Non-linear $\sigma$-models, JHEP 05 (2008) 045 [arXiv: 0802.1659] [INSPIRE].

[5] C.M. Hull and B. Julia, Duality and moduli spaces for timelike reductions, Nucl. Phys. B 534 (1998) 250 [hep-th/9803239] [INSPIRE].

[6] M. Günaydin, A. Neitzke, B. Pioline and A. Waldron, BPS black holes, quantum attractor flows and automorphic forms, Phys. Rev. D 73 (2006) 084019 [hep-th/0512296] [InSPIRE].

[7] G. Bossard, H. Nicolai and K.S. Stelle, Universal BPS structure of stationary supergravity solutions, JHEP 07 (2009) 003 [arXiv:0902.4438] [INSPIRE].

[8] W. Chemissany, P. Fré, J. Rosseel, A.S. Sorin, M. Trigiante et al., Black holes in supergravity and integrability, JHEP 09 (2010) 080 [arXiv:1007.3209] [INSPIRE].

[9] S. Ferrara and S. Sabharwal, Quaternionic Manifolds for Type II Superstring Vacua of Calabi-Yau Spaces, Nucl. Phys. B 332 (1990) 317 [INSPIRE].

[10] D.V. Alekseevskiū, Classification of quaternionic spaces with transitive solvable group of motions, Izv. Akad. Nauk SSSR Ser. Mat. 39 (1975) 315.

[11] S. Cecotti, Homogeneous Kähler Manifolds and T-Algebras In N=2 Supergravity and Superstrings, Commun. Math. Phys. 124 (1989) 23.

[12] B. de Wit and A. Van Proeyen, Special geometry, cubic polynomials and homogeneous quaternionic spaces, Commun. Math. Phys. 149 (1992) 307 [hep-th/9112027] [INSPIRE].

[13] V. Cortés, Alekseevskian spaces, Differential Geom. Appl. 6 (1996) 129.

[14] V. Cortés, J. Louis, P. Smyth and H. Triendl, On certain Káhler quotients of quaternionic Káhler manifolds, Commun. Math. Phys. 317 (2013) 787 [arXiv:1111.0679] [inSPIRE]. 
[15] V. Cortés, C. Mayer, T. Mohaupt and F. Saueressig, Special geometry of Euclidean supersymmetry. 1. Vector multiplets, JHEP 03 (2004) 028 [hep-th/0312001] [INSPIRE].

[16] D.V. Alekseevsky and V. Cortés, Classification of pseudo-Riemannian symmetric spaces of quaternionic Kähler type, in Lie groups and invariant theory, Amer. Math. Soc., Providence, RI, 2005, Amer. Math. Soc. Transl. Ser. 2213 (2005) 33.

[17] V. Cortés, P. Dempster, T. Mohaupt, and O. Vaughan, Special Geometry of Euclidean Supersymmetry IV: hypermultiplets and local c-maps, to appear.

[18] G. Clement, The Symmetries of five-dimensional minimal supergravity reduced to three dimensions, J. Math. Phys. 49 (2008) 042503 [Erratum ibid. 49 (2008) 079901] [arXiv:0710.1192] [INSPIRE].

[19] D. Gaiotto, W. Li and M. Padi, Non-Supersymmetric Attractor Flow in Symmetric Spaces, JHEP 12 (2007) 093 [arXiv:0710.1638] [INSPIRE].

[20] A. Bouchareb, G. Clement, C.-M. Chen, D.V. Gal'tsov, N.G. Scherbluk et al., $G_{2}$ generating technique for minimal $D=5$ supergravity and black rings, Phys. Rev. D 76 (2007) 104032 [Erratum ibid. D 78 (2008) 029901] [arXiv:0708.2361] [INSPIRE].

[21] M. Berger, Classification des espaces homogènes symétriques irréductibles, C. R. Acad. Sci. Paris 240 (1955) 2370.

[22] M. Berger, Structure et classification des espaces homogènes symétriques à groupe d'isométries semi-simple, C. R. Acad. Sci. Paris 241 (1955) 1696.

[23] A.S. Dancer, H.R. Jørgensen, and A.F. Swann, Metric geometries over the split quaternions, Rend. Sem. Mat. Univ. Politec. Torino 63 (2005) 119.

[24] M. Krahe, Para-pluriharmonic maps and twistor spaces, in Handbook of pseudo-Riemannian geometry and supersymmetry, Eur. Math. Soc., Zürich, 2010, IRMA Lect. Math. Theor. Phys. 16 (2010) 497,

[25] V. Cortés, P. Dempster, and T. Mohaupt, Time-like reductions of five-dimensional supergravity with vector multiplets, to appear.

[26] G. Compere, S. de Buyl, E. Jamsin and A. Virmani, G2 Dualities in D $=5$ Supergravity and Black Strings, Class. Quant. Grav. 26 (2009) 125016 [arXiv:0903.1645] [INSPIRE].

[27] G. Compere, S. de Buyl, S. Stotyn and A. Virmani, A General Black String and its Microscopics, JHEP 11 (2010) 133 [arXiv:1006.5464] [INSPIRE].

[28] V. Cortés and T. Mohaupt, Special Geometry of Euclidean Supersymmetry III: The Local r-map, instantons and black holes, JHEP 07 (2009) 066 [arXiv:0905. 2844] [INSPIRE].

[29] A. Sahay and A. Virmani, Subtracted Geometry from Harrison Transformations: II, JHEP 07 (2013) 089 [arXiv: 1305.2800] [inSPIRE].

[30] A. Onishik and È. B. Vinberg eds., Lie groups and Lie algebras, III, in Encyclopaedia of Mathematical Sciences, vol.41, Springer-Verlag, Berlin, 1994. 